\title{
DIGITAL INTIMAGIES
}

AND LGBT+ YOUTH:

CELEBRATION, EQUITY AND SAFETY

Rachel Thomson ${ }^{1}$, Sara Bragg ${ }^{2} \&$ Kate O'Riordan ${ }^{1}$

with Khaliden Alsaleh, Rosie Gahnstrom and Ester McGeeney
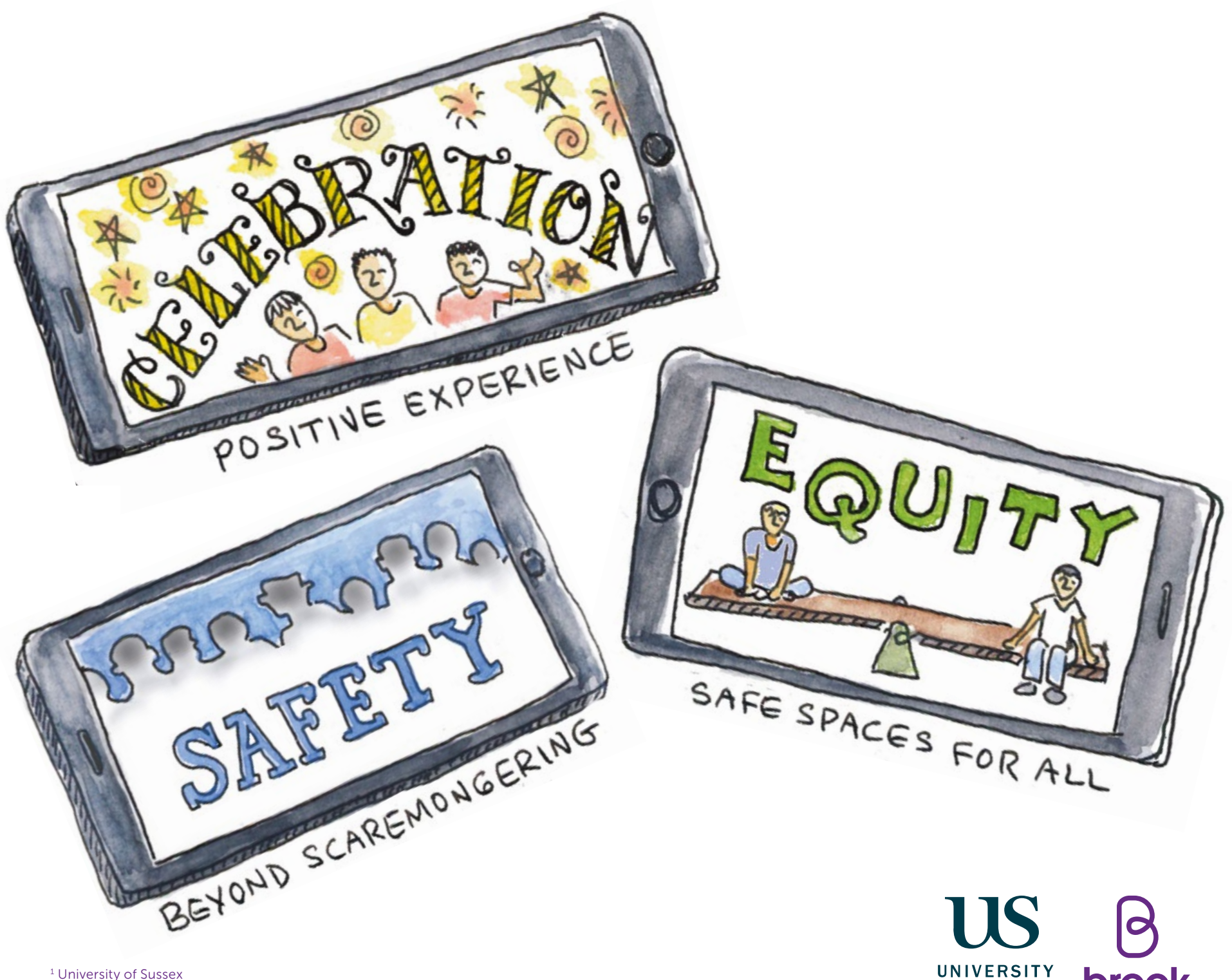
Published by University of Sussex Library, Brighton, September 2021.

ISBN: 978-0-9957862-6-4 DOI: https://dx.doi.org/10.20919/9780995786264

Text copyright 2021 Rachel Thomson, Sara Bragg \& Kate O'Riordan except where otherwise noted.

Illustrations copyright 2021 Michi Mathias.

This work is licensed under a Creative Commons Attribution 4.0 International License.

How to cite: Thomson, Rachel, Bragg, Sara, \& O'Riordan, Kate (2021) Digital Intimacies and LGBT+ Youth:

Celebration, Equity and Safety. Brighton: University of Sussex 
Executive Summary

Chapter 1: Introduction

Chapter 2: Methodology

Research Design and Methods

Strand 1: Reviewing the field

Strand 2: Focusing on young people

Approach to analysis

Definitions and demographics

Samples:

Locations

Limitations of the research

Ethical considerations

Identifiers for data

Chapter 3: Celebration

Self-making in a heteronormative world

Digital intimacies

Generation gaps and alliances

Conclusions

Chapter 4: Equity

The spaces for equity

Different and the same

A space within a space

The challenges of inclusion

Home is where the heart is?

Conclusions

Chapter 5: Safety

Recognising risk

Creeps and bullies

Risk is normal

From scaremongering to safeguarding

Learning safety

Conclusions

Chapter 6: Summary findings and areas for action 36

Summary findings

Celebration $\quad 36$

Equity $\quad 36$

Safety $\quad 36$

Areas for action $\quad 37$

Inclusive Relationship and Sex(uality) Education (RSE) 37

Inclusive online safety

Resourcing anti- harassment strategies $\quad 37$

Demanding more from digital media providers

Actively supporting parents and carers

Creating and networking safe spaces

Supporting youth community spaces

Supporting creativity/world-making

$\begin{array}{ll}\text { Outreach and exchange } & 37\end{array}$

Acknowledgements: $\quad 38$

$\begin{array}{ll}\text { Appendix 1: Glossary } & 39\end{array}$

$\begin{array}{ll}\text { Appendix 2: Practice survey } & 41\end{array}$

$\begin{array}{ll}\text { References } & 43\end{array}$ 
"Digital Intimacies and LGBT+ youth" was commissioned by Brook, the wellbeing and sexual health charity for under-25s. It builds on the insights of its earlier report Digital Romance (McGeeney and Hanson 2017) which researched young people's digital intimacy practices. For this report, we revisited survey and focus group data specifically from LGBT+ participants in that research. Additionally, we reviewed academic literature and gathered new data from LGBT+ youth groups, youth work practitioners and teachers, and parents / carers. Our research took place during the period of lockdowns due to Covid-19. These induced rapid shifts in perceptions of the online and in youth work practices, which have shaped our analysis here.

The report summarises its findings under the headings of:

\section{Celebration}

We acknowledge the positive experiences that LGBT+ youth have online. We recognise LGBT+ youth as engaged creatively in diverse practices of community-building across many digital and offline spaces. Although there may be some (real and imagined) generational gaps in appreciating the value of digital cultures, we describe how youth work professionals rapidly and flexibly adapted their work with young people to online spaces under lockdown. Evidence that youth work practices were in turn able to positively impact some young people's online relationship-building shows the value and vitality of cross-generational work and spaces.

\section{Equity}

We acknowledge the particular challenges faced by LGBT+ youth in a heteronormative world, which require specific resources to ameliorate. Some of these resources - especially for LGBT+ friendly youth services and other provision - are inadequate and / or under threat. Young people in our research looked particularly to schools to create safe and inclusive spaces including through relationships and sexuality education. We also draw attention to the responsibilities of platform providers, since many features of internet architecture prioritise commercial imperatives above privacy, preventing online harassment, or access to information.

\section{Safety}

We acknowledge the particular issues faced by LGBT+ youth in forging relationships and identities and their need for support. These intersect with many different aspects of identity. However we argue that an overemphasis on risk can be alienating and counterproductive, especially if it comes at the expense of more positive representations. Risk-taking is a necessary part of growing and learning. Managing risk should be seen as a lifelong project for all of us, constantly revisited, rather than something that can be avoided entirely or achieved at a single point in time.

We conclude by offering a set of recommendations for practice relevant to funding bodies, campaigners, youth work professionals, schools, families and carers. These relate to:

- Inclusive Relationships and Sex(uality) Education

- Inclusive online safety

- Resourcing anti-harassment strategies

- Demanding more from digital media providers

- Actively supporting parents and carers

- Creating and networking safe spaces

- Supporting youth community spaces

- Supporting creativity/world-making

- Outreach and exchange

Brook's own Summary Report of the findings below can be found on its publications and reports page. 


\author{
In 2017 Brook published Digital Romance, a research \\ report exploring young people's use of technology \\ in their romantic relationships and love lives. Brook \\ is the leading sexual health and wellbeing charity for \\ under 25s. Brook supports 235,000 young people \\ each year through clinical services, education and \\ wellbeing work.
}

Digital Romance by Ester McGeeney and Elly Hanson drew on a UK-wide survey of over 2000 14-17 year-olds and talkbased methods with around 80 participants. It captured how digital technologies have become integral to the relationship cultures of contemporary young people, including practices for meeting, flirting and getting intimate, breaking up and falling out.

The diverse sample included young people who identified with different sexual identities (including lesbian, gay and bisexual) and different gender expressions (including cis, nonbinary and trans). The original research identified $L G B T+{ }^{1}$ youth as having some specific needs and experiences, within the broader category of young people.

In relation to $\mathrm{LGBT}+$ young people specifically, the 2017 Digital

Romance research found that:

- LGBT+ young people described more benefits to digital technology but experienced more online risks;

- Higher proportions of LGBT+ young people had met a partner or asked someone out online;

- LGBT+ young people were more likely to often meet people in person who had deceived them online;

- Participants did not feel that they received adequate education on positive relationships;

- Participants were clear about the need for educators to include and address the needs of LGBT+ young people when delivering education around online safety and positive relationships.

The inadequacies of relationships and sex education (RSE ${ }^{2}$ ), and LGBT+ young people's vulnerability to harassment in schools, were echoed in other contemporaneous research. Stonewall's 2017 School

Report $^{3}$ revealed that just 1 in 5 LGBT+ young people had been taught about safe sex in relation to same sex relationships and that 45 per cent of LGBT pupils - including 64 per cent of trans pupils - reported being bullied for being LGBT in Britain's schools. In 2018, the Government's National LGBT+ Survey ${ }^{4}$ also found that the education system was not preparing LGBT+ young people for later life. Only $3 \%$ of respondents said they had discussed sexual orientation and gender identity at school, be that during lessons, in assemblies or elsewhere.

\section{Brook commissioned further} research in late 2019 and early 2020. The aim was to identify the key challenges, opportunities and resources for practice and policy development in this area: a roadmap for supporting LGBT+ young people navigate a digital landscape for the making of friendships and romantic and sexual relationships.

This research was to be conducted through:

- Secondary analysis of the original Digital Romance research data;

- Literature analysis to contextualise the findings in a wider body of scholarship;

- Consultation with reference groups ${ }^{5}$ of LGBT+ young people accessed through youth work settings;

- Consultations with stakeholder groups including parents of LGBT+ youth, teachers, and specialist youth work and health providers. 
Then Covid-19 and lockdown changed everything. Fieldwork was interrupted and eventually moved online, and social distancing transformed the everyday lives of us all. The dynamics of the research field and policy context shifted as face-to-face interactions were curtailed and digital platforms became the primary medium for all forms of communication. We continued to research during this period, recognising the enormity of these events for the topic of our enquiry. We wanted to know how organisations working with LGBT+ youth were responding to these challenges, how lockdown reframed questions of risk and safeguarding and how generational gaps in knowledge and understanding might be bridged by this unexpected shared experience of enacting intimacies and sociality at a distance (Hanckel \& Chandra 2021; Watson et al 2021).

In Chapter 2 we provide more detail concerning the methods used in this project and the timeline of the research and consultation process. Our initial frustrations about delay and worry that the research would be 'obsolete', gave way over time to a new sense of perspective. After a year of living with a pandemic, we understood that we were better able to untangle and separate the enduring issues from the dynamic and contextual 'noise' that surrounds digital technologies and youth.
This research was also carried out in a new policy context where the responsibilities of commercial platforms are being addressed in the Online Safety Bill and new agenda - for statutory relationships, sex and health education and for tackling sexual abuse and harassment in physical or virtual spaces ${ }^{6}$ - are framing the responsibilities of schools. This is also a climate where arguments for equality need to be remade, recognising generational tensions and how 'digital' is now an integral part of new expressions of gender and sexuality?. In a post-pandemic world we will never again think so simplistically about online and offline worlds. Many of us have now experienced the problems and pleasures of managing an identity online and struggled with controlling the collapses of context between public and private personas. These are now everybody's problems, not just an aspect of youth or queer culture.

A large body of material was generated in the process of this research and we have had to be highly selective in what we present in this report. We have tried to be careful and consistent with how we label each item of evidence or testimony, be it a statistic from the survey, a quotation from a group discussion, or a one-to-one interview conducted in person or online. Our aim is to provide a framework for understanding what digital romance may mean for LGBT+ youth at this moment in time and to outline intersecting fields of action where it may be possible to use insights to inform and improve practice.
The report is structured through three themes which emerged in the study:

Celebration: digital culture is a vital space of LGBT+ community and queer world-making.

Equity: continues to be a struggle for LGBT+ youth faced with the everyday realities of sexism, heteronormativity, abuse, harassment, and discrimination, which can be impacted particularly by the commercial structures of digital culture.

Safety: risk-taking is a normal part of teenage- and young adulthood, but it is experienced by LGBT+ youth in distinct ways that also intersect with other aspects of identity including ethnicity, religion, and neurodiversity, and socio-economic factors.

In each section we provide context and outline the findings from the research drawing out their implications. In the final chapter we summarise the findings of the report and the areas for action needed to ensure we develop effective and appropriate education interventions where LGBT+ young people are full, equal and safe participants in online activity and communities, and are given the opportunity to develop the knowledge, skills and attitudes to stay safe online.

\footnotetext{
${ }^{1}$ Following Brook's house style, the terminology LGBT+ is used consistently in this report irrespective of whether it was used in the material cited. The inclusive term 'queer' is also used at times, reflecting key ideas from the literature or young people's own usage of the term.

RSE (Relationships and Sex Education) is used in this report to reflect the English context in which the research was carried out. In Wales the new compulsory subject is called Relationships and Sexuality Education. Globally 'comprehensive sexuality education' is widely used, and may better reflect the aim of addressing the whole person including their sexuality, behaviour and identity rather than sex as a discrete topic.

Stonewall School Report 2017

${ }^{4}$ The National LGBT Survey 2018

Reference groups with young people were carried out face to face and are identified by location (urban, small city, rural) and age group (either under 16, 16-25 or mixed).

Stakeholder groups, which were carried out virtually, are identified by membership. All material is identified by the year in which it is created.

6 The Department for Education has issued guidance for statutory RSE in schools in England. The Schools Inspectorate has published Review of sexual abuse in schools and colleges (Ofsted, 2021).

(Ofsted, 2021).
See for example the digital sexual cultures feminist research and engagement consortium.
} 


\section{Here we present an overview of the strands of the research for the project including research questions and method.}

\section{Research Design and Methods}

Strand 1: Reviewing the field A literature and practice review were conducted to explore the following research questions:

i. What does previous research tell us about the needs and experiences of LGBT+ youth use of online media for digital romance (online dating cultures and sexual expression)?

ii. How does the field define current priorities, needs and resources?

\section{Literature review \\ (Dec 2019-Apr 2020)}

A focused literature review explored social research into LGBT+, youth and online sexual cultures and digital intimacies, evaluations of sexual health interventions aimed at LGBT+ youth in general, and evaluations of interventions aimed at online safety and wellbeing for LGBT+ youth. It involved consulting with leading academic experts in the field of digital/online LGBT+ sexual cultures and sexual health interventions ${ }^{8}$, and searching for literature (2009-2020) via online academic databases.

Practice review (Feb-Mar 2020) In order to establish the concerns and priorities of those working with young people, including information about resources, interventions and materials already in use and priorities for the future, an online questionnaire was designed collaboratively between the Sussex research team and Brook, which was circulated as widely as possible to practitioners working with children and young people, through Brook networks between late February and March 31st 2020 (see appendix 2).

Strand 2: Focusing on young people To help us focus on young people, we carried out a secondary analysis of the original Digital Romance data and reference group consultations with young people in order to address the following research questions:

i. How can we characterise and understand the particular needs and experiences of LGBT+ young people in relation to digital romance (online dating cultures and sexual expression)?

ii. To what extent are findings that formed part of the Digital Romance 1.0 study still valid and relevant? What are the gaps?

iii. How has the landscape changed since 2017 in relation to (a) online dating for LGBT+ young people and (b) LGBT+ young people and online safeguarding?

\section{Secondary analysis (Dec 2019-Jan 2020)}

The first of these questions was addressed through a secondary analysis of $\mathrm{LGBT}+$ responses within the 2017 Digital Romance dataset, including both closed and open survey questions, and qualitative focus groups and interviews.

\section{Consultations with young people (Feb-March 2020)}

In order to check the continued relevance of these findings and to deepen understanding, four face to face reference group consultations with LGBT+ young people were set up during 2020 . These groups took place in three youth work settings: urban, small city and rural. Apart from one group with young people aged under 16 , the groups were mixed in terms of age (14-25). Emergent findings from the secondary analysis were rephrased as prompts for discussion utilising a game format, 'youth values continuum', that encouraged debate and equal participation ${ }^{9}$.

\section{Strand 3: Informing} future developments To help us understand how Brook might contribute to the development of new resources and interventions we posed the following research questions: 
i. What research messages can be identified for key stake-holding groups: (a) teachers (b) parents (c) specialist youth workers and health workers?

ii. What new areas of development are a priority?

iii. How can Brook best meet the needs of stakeholders?

iv. How has the experience of Covid-19 and associated isolation policies affected this set of issues?

\section{Stakeholder groups (Nov-Dec 2020)}

This stage of the project involved a series of online events with stakeholder groups. These were advertised through Brook's extensive networks and led by Brook development workers, drawing on emergent findings briefings developed by the research team. Discussions were recorded and summaries made of key themes and findings. In order to capture the impact of Covid-19 and social distancing on key providers working with LGBT+ youth, one to one interviews were undertaken by the Sussex research team in April 2020.

\section{Approach to analysis}

The staged approach of the research required analysis to inform each successive stage. A working paper (Alsaleh et al. 2020) captures the analysis for each element of the research, which led to our identification of the three conceptual foci for this report: celebration, equity, and safety. Here we present a synthesis of findings from across the research and development process. Those interested in the detail of each element and a fuller exposition of the methodology should consult the working paper.

\section{Definitions and demographics}

LGBT+ is a diverse group including different sexual identities (gay, lesbian, bisexual) and different gender expressions (cis, non- binary and trans). In the original 2017 Digital Romance survey, of all respondents, $64 \%$ identified as female, $31 \%$ male, $3 \%$ non-binary, $3 \%$ identified as transgender and $3 \%$ unsure of their gender identity. In terms of sexuality, $18 \%$ identified as bisexual, 6\% gay/ lesbian and $71 \%$ heterosexual. In the reporting of open questions from the survey we have included key demographic information including self identifications for gender, ethnicity and sexuality.

In the new research all young people were accessed through specialist youth services or special nights for LGBT+ youth, which in one setting enabled us to speak separately to an under-16 group. The question of self-definition was often a live and unresolved one for the young people involved in the research and we did not request that they self-define as a condition of being involved in the study. In line with agreements with participants these voices have been presented under the LGBT+ umbrella label. In general, the voices of young people who are not involved in or accessing services are underrepresented in this part of the research, and this may in turn reflect other intersectionalities including social class, ethnicity and religion.

\section{Samples}

The research reported on here draws on:

a) The original Digital Romance sample of 14-25 year-olds (2017), focusing on the $500+$ questionnaire responses and 18 individual contributions to focus groups and/or interviews by young people who identified at LGBT+ (approximately $25 \%$ of the overall sample);

b) New face to face reference group discussions with LGBT+ young people (2020/21), comprised of four face to face group discussions and two individual interviews, one of which was online (a total of 18 young people contributing overall);

c) Stakeholder groups including

- Practitioners: which includes a survey of teachers and service providers ( $n=183)$, two online discussion groups with teachers and service providers (16 people), and individual interviews with two specialist service managers reflecting on the impact of lockdown.

- Parents and carers virtual discussion group (7 people).

In terms of the two waves of research this report draws on the voices of over 500 young people identifying as LGBT+ and over 200 engaged adults including educators, service providers and parents.

\section{Locations}

Location is an important factor shaping the experiences of LGBT+ young people, including access to services and community. In both the original Digital Romance study (2017) and the new research we made efforts to ensure some diversity in locality in order to capture the importance of place, yet we have not named these as part of our commitment to confidentiality. In the original Digital Romance 2017 study, qualitative research took place in rural/suburban locations in southwest and southeast England. Localities of the 2020-1 research include urban areas in the North and South as well as a small city and a rural town again in southwest England. Virtual methods drew participants from a range of areas across the UK. We do not make claims about specific places in this research, nor claim generalisations from the research to all places in the UK, however, following the literature, we have endeavoured to ask about place as an important dimension of young people's experiences. 
Limitations of the research

Neither the original Digital

Romance research nor the followup research for this report can be understood as nationally representative studies. Participants were self-selecting and access for the qualitative part of the research has been through specialist services and networks. In the original Digital Romance survey research LGBT+ respondents were overrepresented in the sample at approximately $25 \%$ rather than the $6.6 \%$ of $16-24$ year-olds estimated to identify as LGB (ONS 2019).

As a whole, the study draws on more than 500 survey responses and 36 contributions to discussion groups or interviews. However, we must assume that the qualitative component in particular underrepresents young people who are not in contact with services and potentially those for whom identifying publicly as LGBT+ is difficult or dangerous. The research also draws on contributions from over 200 adults engaged in education or support. Again, our use of existing networks means that those responding were likely to be interested, engaged and well-informed in relative terms. In our reporting we have been careful to show the limits of the findings and the specificity of the data reported both in terms of when the material was collected and how it was generated.

\section{Ethical considerations}

This research invited young people to talk about intimate experiences and we were careful to ensure that our approach was ethically robust. We paid attention to informed consent, ensuring that participants understood and could reflect on the nature of the investigation including their ability to withdraw from the research and to remove their data. We also attended to confidentiality, explaining both the limits of anonymity and our commitment to protecting individual identities and localities in the reporting of findings. Individual level detail on gender, age and sexuality is only provided for the 2017 questionnaire responses.

In line with the original 2017 Digital Romance study, qualitative research participants were invited to choose their own 'fake names' for the reporting of their words and we have changed or omitted information that would undermine anonymity. We agreed with young people in the qualitative phase that they would not need to declare or share gender or sexual identities, so materials arising from these groups are identified in general terms relating to location and the age range of the group. For all qualitative work the research team worked within the safeguarding policies of the organisations that hosted the research, something made clear in information provided to participants and ground rules for discussions. Where disclosures of potentially harmful and/or illegal activity were made within the research, we worked with workers from the host organisations to ensure both that they were aware of the disclosure and that the young person was being appropriately supported. The research team are all experienced youth researchers with a track record of working in areas of sexual health and education. Our commitment to participation meant the use of creative methods that ensured turn-taking and different ways of giving voice within the research.

The research design was submitted for ethical review by the University of Sussex Social Science and Arts CREC (ER/ RT219/6) and the approach was interrogated and approved. The approach adopted in this research is consistent with the guidelines for ethical practice of the British Sociological Association and the 5 key principles of Government Social Research Ethics.

\section{Identifiers for data}

In the report we use the following identifiers when presenting findings and quotations, providing relevant further details as appropriate and possible (e.g location, age, role).

\section{Original Digital Romance data} Survey 2017 [quotations including self reported age, gender and sexual orientation ${ }^{10}$ ] Focus group 2017

Newly generated data

Practitioner survey 2020

Reference groups 2020 [urban/ small city/rural and either under 16 , 16-25 or mixed age] Consultation groups [parents, teacher and specialist youth work and health providers] 2021

Interviews [identified either as service provider or young person] 2021

All personal names used are pseudonyms and were chosen by participants.

\footnotetext{
${ }^{8}$ Academic experts consulted included: Ben Light (University of Salford), Jessica Ringrose (UCL Instutute of Education). Chris Bonell and Rebecca Meiksin (London School of Hygiene and Tropical Medicine). Olu Jenzen (University of Brighton), colleagues in the Centre for Innovation and Research in Childhood and Youth at the University of Sussex.

${ }^{9} \mathrm{http}$ ://www.restore.ac.uk/inventingadulthoods/capturing/research_time/youth_values/values_8.shtml

${ }_{10}$ Demographic details are included for comments from the 2017 survey only.
} 


\section{CHAPTER 3: \\ CELEBRATION}

In this section of the report, we look at the importance of digital spaces for the making of queer culture and the celebration of community. We centre the experiences of young people growing up in diverse families, communities and localities and attempting to make sense of themselves, often through trial and error. In telling this story we cover the following findings from the study: the necessity of digital practices for self-exploration and the making of community; the challenges of visibility; the importance of queer spaces and the potential for LGBT+ young people to be creators as well as consumers of culture.

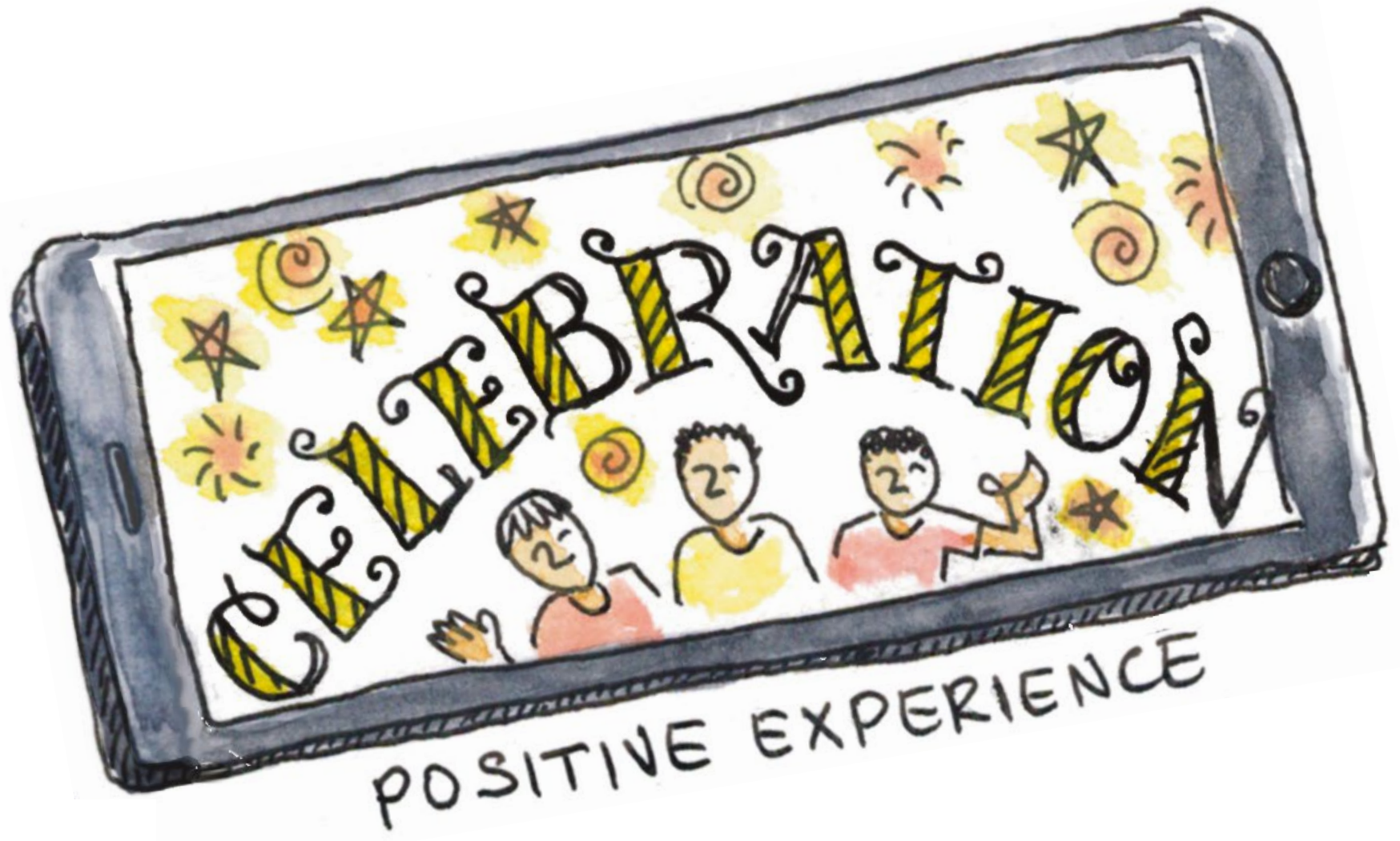




\section{Self-making in a heteronormative world}

The term 'heteronormativity' was introduced by Michael Warner in 1991 to encourage a shift in critical attention, away from a focus on LGBT+ (whether as difference, problem or 'pathology' such as an illness or a crime) to that which is taken for granted and naturalized as the norm heterosexuality. Over time this way of thinking has helped to address the stigmatisation of LGBT+ identities and to question polarized and mutually exclusive ideas of masculinity and femininity. The current consensus ${ }^{11}$ is that 'heteronormativity is what makes heterosexuality seem coherent, natural and privileged. It involves the assumption that everyone is "naturally" heterosexual, and that heterosexuality is an ideal', superior to other sexualities.

\section{Heterosexuality continues to} be taken for granted in many if not most cultural spaces. Not fitting with the norm may still involve struggling with negative stereotypes that are both internalized and come from others (that feelings and desires are morally wrong or signs of illness) - in ways that take a toll on mental health (McDermott et al. 2019). Questions of 'who am I?' and 'where do I fit?' were urgent questions for the young people in this research and before we explore questions about online dating and romance, we first need to consider the importance of self-exploration and 'coming out'. Across the different strands of the research, we gained a strong sense that online spaces were important to LGBT+ young people as a way of meeting and getting to know others away from local peer, family or faith groups. As one of the respondents to the 2017 survey observes:

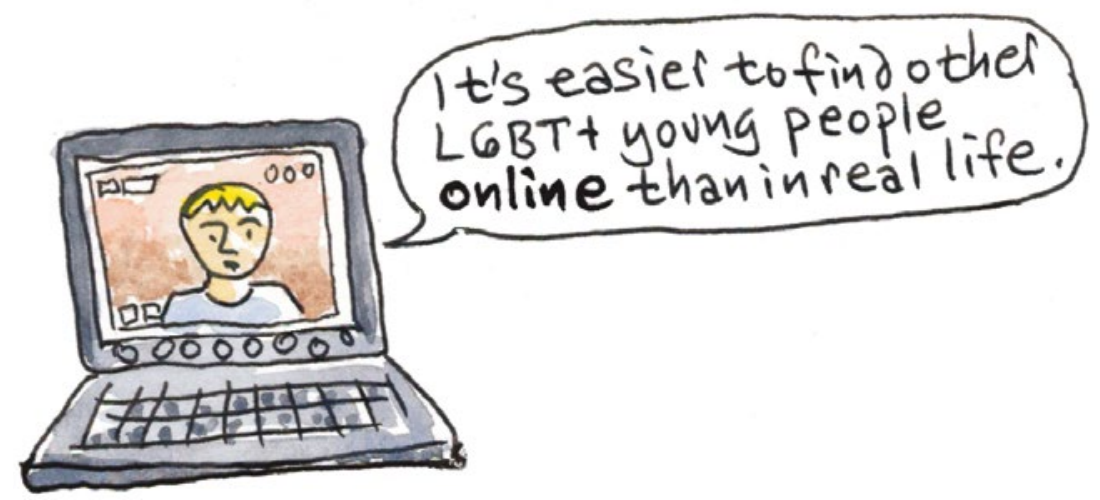

Being a young queer person, it can often be difficult to meet other queer people in "real" life to form relationships. This is much easier in the digital world. [survey 2017, aged 22, female, White British/ English, lesbian]

Online spaces can be vital for making friends. The following discussion in an under-16s LGBT+ group captures how they use different platforms in order to explore and share aspects of identity:

Maisie: I made a lot of friends online [...] when you think about it, the good kind of outweighs the bad a bit more, like not completely but like to a certain extent and you know like it's much easier to find a community where you belong online because you can literally just like go on Google or something and just yeah whereas sometimes in real life it's a lot Clock: - more difficult

Lamp: I'm a lot more open on social media than I am in real life... More my true self rather than a different person Moca: I'm a lot more energetic when it comes to like talking to people online - [urban, under 16 reference group, 2020]

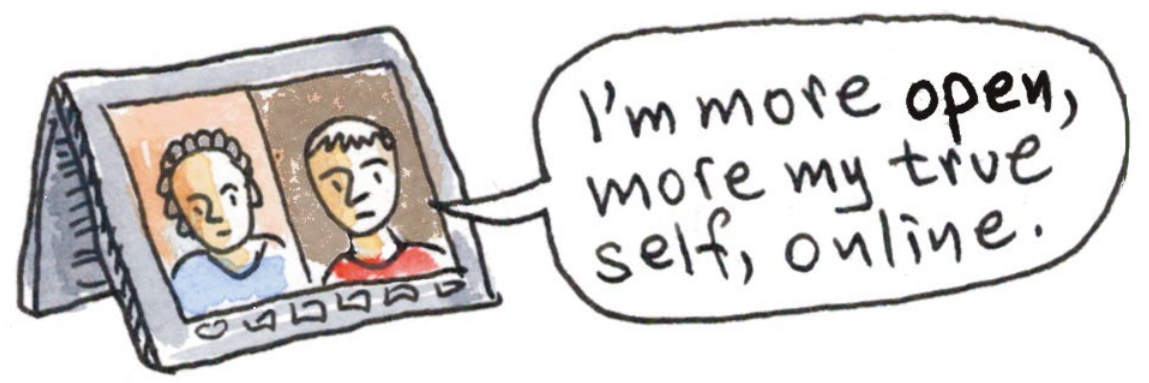

The wider research literature suggests that LGBT+ youth make use of digital spaces to compensate for lack of connection, community, validation or support in offline contexts. This is often positive for social support, developing LGBT+ identities (DeHaan et al., 2013; Harper et al., 2015; McGeeney and Hanson, 2017), finding information or partners and increasing wellbeing in online contexts of relative safety (Selkie et al., 2020). Marston (2019) describes digital platforms as enabling 'rehearsals' of samesex 'identity, friendship, coming out, intimate relationships, sex and community', easing and accelerating any coming out processes.

These insights are echoed by the young people attending the urban 16-25 group who reflect on how isolated young queer people can be, arguing that although online spaces may be insufficient on their own for the making of community, they can also be liberating. Sam notes: 
A lot of the LGBT people can be in places where they don't really know anyone else that's in the same position as them and it can be isolating. So, you can talk to as many people in your life as you want but you still feel very lonely and that online gives them the chance to meet other people that have similar experiences. [urban, 16-25 reference group 2020]

Isolation can be amplified by additional factors which intersect with gender and sexuality. In the same group Jimmery talks about accessibility:

If you're a young carer, if you are disabled and you can't maybe get to places or don't have the money to travel to LGBT safe spaces like this one... having an online community can literally save lives.

Online community is never straightforward: in this group another participant suggests that online interaction 'can increase the isolation by avoiding the interaction on a face to face human level - which can produce a greater anxiety and greater isolation'.

Yet the online is not simply a space for 'rehearsal', it has validity and meaning in its own right. Gray's (2009 a, b) study of rural US LGBT+ youth shows the essential role online media play in youth queer identity-crafting. It also demonstrates the nuanced ways young people rework digital source material such as avatars, characters or platforms to create identities suited to their own local spaces - and thereby build 'liveable lives' (Browne et al., 2021). Downing (2013) notes how non-heterosexual young people manage and negotiate the connections between virtual and material spaces in their everyday lives. In our research young people living in a rural area emphasise how online spaces allow them to feel a sense of belonging to a broader LGBT+ community:
Harley Quinn: I think it's mainly to feel part of a community if I'm honest

Lady Gaga: Because you can find and feel part of a community but sometimes it can be hard to find

like a right space to go to

Moderator: So, is it easy to find other LGBT+ young people online? Lady Gaga: it's easier than trying to find them in real life [...]

Harley: they haven't come out, or they don't actually know what they are yet and are questioning - and like I know there's a few apps where LGBT+ people can just go on there and chat to each other [rural, mixed age reference group, 2020]

Digital platforms are diverse and provide different ways for young people to meet and interact. For example, gaming platforms may allow young people to explore sexuality through avatars or other types of alternate personalities, perhaps mattering more to LGBT+ youth than heterosexual peers. Other platforms may provide ways of participating in various activisms (Keller 2019). Practices such as 'lurking' (Berriman and Thomson, 2015) - being present without being visible - can be understood more actively for LGBT+ young people. They enable a form of listening that is social and participatory and an especially important form of engagement for young people who may not usually feel safe engaging in discussions about gender and identity.

One of the young people in the rural mixed age reference group goes on to explain that he met his best friend while online gaming, while also noting some more difficult aspects of community spaces (something we discuss in more detail in chapter 5):

It doesn't mean that I was looking for an LGBT person, it just, she happened to be that and I think, yeah some of us do look for other LGBT people because you know it feels part of the community but not for me personally because I feel like the community is somewhat a bit toxic.

We find similarities here with Hanckel and colleagues who describe the LGBT+ youth in their study as engaging in 'queer-world building' - finding, building and fostering support beyond the self in ways that contribute to their health and wellbeing (Hanckel et al., 2019, Bryan, 2019). An attentiveness to queer-world building practices expands attention from dating apps or sexual health information, towards a much wider network of connected sites, users and content (hashtags, memes, blogs etc). Maisie explains that she follows:

A lot of LGBT accounts, on Instagram and things like that. Sometimes they post like stupid gay memes that everyone can laugh at, or sometimes they post like actual support which is obviously really nice. [urban, under 16 reference group 2020]

In the same group Moca reflects on how LGBT+ community evolves on Twitter, taking shape in some cases through challenging homo/ bi/transphobic tweets:

There is a very prominent community of LGBT youth or like LGBT adults because whenever you go on like a controversial tweet there will always be those like um I don't know what you call them ... Trump supporters. And like you see them but at the same time you also see those comments, like those replies to those comments were, like "No, absolutely not. you're like completely wrong" and it's just like "yes, we need this".

In the research literature Marston (2019: 280) emphasises the 'lived and deeply felt sociality of everyday encounters with selfies, memes, gifs, emojis, algorithms, and site architecture' for LGBT+ young people in sharing and 
circulating feelings. Jenzen and Karl (2014) also argue that comics, music and various forms of online popular fan culture are equally important for engagement with issues of identity and trans culture. Our research findings align with theirs, similarly showing how young people are countering discrimination, harassment and abuse by creating new symbolic languages for identity, community and activism through memes, DIY vlogs, widgets, cat gifs, fan mashups, unicorns farting rainbows and tagging.

Arguably, queer digital culture allows users to curate and control their interactions, at least to a certain extent. Our participant Nora explains that as a queer South Asian woman, she struggles to be out with her family. Yet she is able to find community in her life at University and online. 'I'm very aware that I live in a very unique queer safe bubble for me. Like, when I am in this bubble I don't experience a lot of like- like when I talk about voting with my parents....' She describes one online support group where 'you send selfies and it's positive and uplifting'. Her words resonate with the research on trans vlogger sites which argues that trans stories can animate and motivate others to dare to be visible or claim an identity as trans (Raun, 2014, Jenzen, 2017).

I find it really inspirational actually, like I see all these trans people putting pictures of themselves out there and people are lifting them up and I find it so brave and I'm like 'these people are amazing' and I can't imagine the lives they had to live. Like, I look at my girlfriend and I'm like... she's incredible. I can't imagine how hard it is. Like I know how hard it is to be queer and the struggle of that like, you know. Realising that you're not who you've been told you were your whole life like, it's crazy right? [Nora, interview, 2021]
Social research increasingly shows how digital platforms and technologies are intimately entangled in daily lives, relationships and practices, rather than being conceptually or practically separable into online and offline (Andreassen et al., 2017; Latimer and Gomez 2019). Community spaces need to be understood as transcending the online/offline divide, with faceto-face spaces in youth clubs or in schools being informed by the online spaces and vice versa.

In a discussion group in the rural area, Sophie captures the subtle ways that communities of cultural interest (for example in anime) may intersect with communities of sexual identity and gender expression:

At my school we have what we call the little gay corner. We all kind of go and it's a whole lot of people. There's our little corner, we have our friendly neighbourhood lesbian and we have two gender neutral people (which is now one because someone has left the school), a trans guy and myself we all kind of sit down and we chat about gay things. It doesn't matter that we're all different kinds of gay, it's all kind of common ground because in the end we all go through the same kind of struggles with people who don't understand us and discriminate against us. There's this common ground. And also they're all really into anime. I don't get that, but they do, so, just go for it. [rural, mixed age reference group 2020]

We return to questions about equity, inclusion and diversity in Chapter 4. However, here we want to emphasise the value given by young people in the study to community spaces (both virtual and face to face), through which they could learn about and contribute to LGBT+ culture and get a rest from the 'normative cruelties' (Ringrose and Renold
2010) of everyday experiences. In Harley's words:

There's not really much of a difference between a lot of the $L G B T+$. Because we all are dealing with the same problems, and we all know how it feels to be discriminated against, to be targets of jokes things like that. And like talking... a group like this is a good way of getting away from all that stigma of secondary school and people being in your face, making jokes about your sexuality and all that.' [urban, under 16 reference group, 2020]

\section{Digital intimacies}

If LGBT+ young people are especially reliant on digital platforms for sociality, meeting and making friends and connecting with community, then it is not surprising that romantic and intimate relationships also have a strong digital dimension which the term 'digital intimacies' captures. In the research literature Miles (2018) notes that the distinctive relationship between queer and digital culture is at least 30 years old, and that there is a complex interaction between queer intimacies and the digital. Gay and bisexual men were early adopters of new technology - and the development of locative media applications has been informed by queer concepts of cruising and community. Digital technologies, he argues, have not only shaped queer male spaces over the past three decades, but increasingly constitute what these spaces are, how they are performed, and who is able to access them (Miles, 2018).

A key finding of the 2017 Digital Romance research was that LGBT+ young people were more likely than cis $^{12}$ and heterosexual peers to have asked someone out online and to have dated someone they had met online (McGeeney $\&$ Hanson 2017:17) One reason may be that LGBT+ youth are more 
likely to socialise and 'date' outside of the locality:

If you're unable to see each other in person very often, it helps you keep connected and lets you still have important conversations and connections with your partner. It helps you feel closer even if there is distance and life obligations that may get in the way of you seeing each other very often. [survey 2017, aged 22, female, White British/English, bisexual]

Less stressful for people with social anxiety, makes long distance relationships easier, you can find people that share interests with you easier.' [survey 2017, aged 18, female, White British/English, bisexual]

Online environments may also be preferred because of the ambiguity and anonymity they offer. The research literature characterises this in terms of spaces where identity is not simply expressed, but created and re-shaped (Cover, 2012, Downing, 2013, Ringrose and Harvey 2017). For example, Cover argues that social networking enables particular and potentially incompatible performances of identity. On one hand these push towards recognisable identities - for example through the creation of 'profiles' which ask for age, gender identity, taste selections (for example an interest in anime), and biographies. On the other hand, the affordances of the media pull us into more ambiguous and relational identities - for example through friend-list maintenance and communication, wall posts, tagging.

The particular affordances of digital communication were commented on by young people in our research who appreciated how online flirting could enable the exploration of emerging sexualities:
I think, like, being able to find people online is, like, an easy way to test the waters....to, like, experiment or to, like, reaffirm your own sexuality and stuff like that. [focus group 2017]

Importantly, this may also allow for an explicit discussion of sexual preferences and practices in a safe way:

It's like an introduction to being able to, like, explore, being able to communicate about what you want in sex with other people. [focus group 2017]

In particular, asynchrony (where communication is not live) and anonymity (where it is not necessary to reveal your face or real name) can make it easier to talk about desires and safety practices.

It can feel more comfortable to talk about boundaries and needs through messages rather than face to face - they have time to process what has been said and consider different aspects. Also good for people with anxiety who can't go out a lot like me. [survey 2017, aged 21, female, White British, bisexual]

Social media helps people talk about things that improve relationships but wouldn't necessarily be comfortable talking about face to face, such as sexual boundaries. [survey 2017, aged 17, female, White British/English, bisexual]

One impact of heteronormativity is the sexualisation of LGBT+ identities - that is, assuming that LGBT+ lives and behaviours are focused around sex, gender and sexuality, such that for example, communications are sexual rather than social. This can make it difficult to separate risky dating behaviours from the work of community belonging. In practice these distinctions can be blurred, just as they are for heterosexual young people. For example, Alan talks about 'repurposing' a hook up app 'in a community way' by making friends with potential sexual partners - bypassing the intention of the platform. In this way mobile hook-up apps are used for 'off-label' functions (not as intended by the design) or 'intimacy practices' (belonging, chat, friendship) as well as a password-protected 'container' for sexual experimentation or pictures (Albury and Byron, 2016).

These underpinning assumptions were addressed directly in this research. The urban 16-25 group discuss the statement 'Using dating and hook up platforms is different to going online for community information or activism' - giving rise to a rich debate. For instance, Gregg welcomes the insertion of HIV education and domestic violence alerts on dating apps. Burt argues that all kinds of digital sociality (platonic, romantic, business, activism) are fluid, while Max counters that dating apps and community forums are as different as 'ying and yang'. Jimmery disagrees, citing examples of both 'people who've engaged in Tumblr subcultures to find a girlfriend', and people who have 'ended up using hook-up apps and making tons of friends'. The message is that there is not a definitive way in which young people move between digital spaces; and there is fluidity between friendships and romantic relationships.

\section{Generation gaps and alliances}

Our consultations and conversations with adult stakeholders captured a strong sense of concern about what young people were doing in online spaces. This was coupled with a perceived generation gap in terms of digital competence and understanding which in turn could amplify concerns. The parents who participated in the 
consultation group wanted to keep their children safe and protect them from harm to which they feared they might be exposed in unmediated online spaces including contact with much older adults (see Chapter 5 - Safety). There was little consideration of the potential for online spaces to provide support, solidarity or fun for their children, coupled with a lack of familiarity with these spaces. The existence of a generation gap was confirmed by young people such as Sophie, who talks about the efforts she has made in order to educate her mother about what it means to 'know' someone online':

I've had to persuade my mum that I know these people online, they aren't out to get me, there are people who just want to be friends. And it's just a foreign concept, she doesn't really understand that. It's like, my parents are, in a word, 'boomers'. They aren't able to relate to the problems of the internet. They'll overreact or they won't understand or emphasise. They'll just be instinctual, that everyone's out to get them, safety dome, bubble around, keep them safe. [rural, mixed age reference group 2020]

The teachers we talked to in this research were also preoccupied by questions of digital risk and online safety (see Ch 5 Safety), and the need for schools and lessons to meet the needs of LGBT+ young people (see Ch 4 Equity). We found little knowledge among these teachers of LGBT+ young people's positive online experiences, their ability to recognise and manage risk or the potential for digital platforms to provide affirming spaces for them. However, they acknowledged the need to learn from LGBT+ young people themselves about the spaces they want and the support they need.

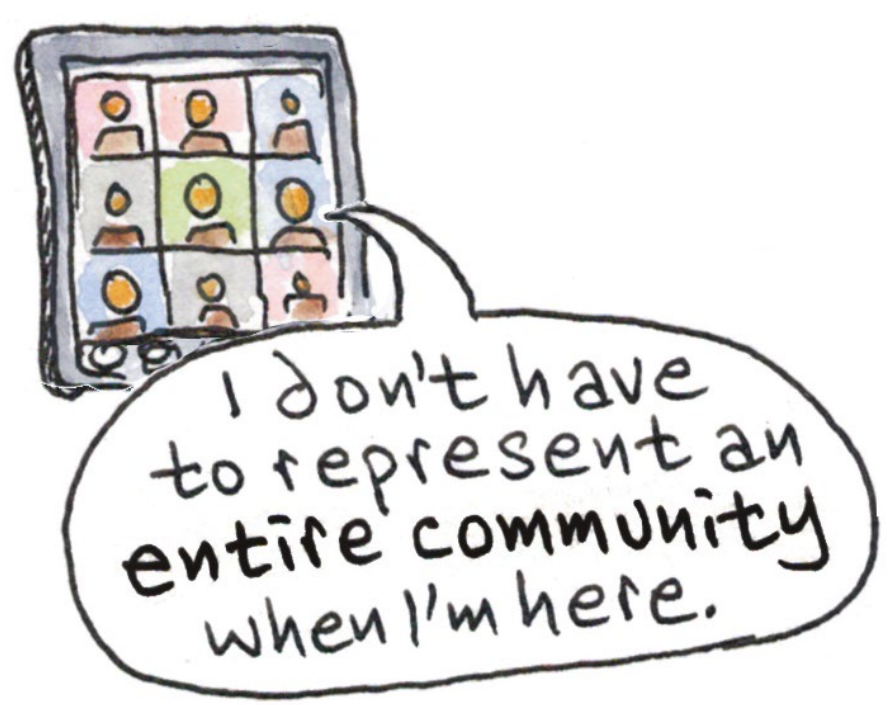

I can come up with things that I think would need in the online space to make them safe, but might not be relevant. So, this is the key area where young people should have more involvement over making those decisions about what that space should look like and what they need. We are often quite removed as adults from how young people are using that online space. So, whether that's just gathering more evidence from young people or young people being more actively involved in creating better education. That comes from respecting young people and giving them an opportunity to be paid for that. [teacher consultation 2021]

LGBT+ professionals working with young people were more attuned to the importance of creating spaces for young people, where they were able to be themselves, have positive role models and feel able to celebrate who they are. One key insight was the importance of spaces where young people don't have to talk about their 'otherness', but can ask questions and look up to LGBT+ adults as role models.

When young people come to our services, being LGBT+ is the least interesting thing about them. They are in a room with others, which allows them not to have to be the sole representative of an entire community, which they often are if they are at school or with their families. [...]. It means they can engage in other areas of their life, not having to think about the fact they are LGBT+. Because they have all of these questions about the world, which in all other aspects of their lives are answered in a cis heteronormative space that highlights they are not this. [specialist youth work and health provider consultation, 2021]

However long you stay in that space, whether you feel happy with being part of a big general group, whether you go on to find a group that more closely reflects you, that's a person's choice. The opportunity for a queer world making in such a heteronormative society is really beautiful and really strong, and really supportive of that. Whether you drop in for half an hour or stay forever. [specialist youth work and health provider consultation 2021]

For most of the adults that we spoke with, these positive spaces were understood to be in-person and face to face. They might exist at school (signalled by visible symbols of inclusivity such as rainbow coloured lanyards) or at special nights in mainstream youth 
clubs or in LGBT+ community venues. Yet positive spaces clearly also exist and can be created online.

We end this chapter with an instructive story from the height of the first UK national lockdown in response to Covid-19. It came from the manager of a LGBT+ youth service who had managed to move the organisation from face to face to virtual provision in a matter of weeks. She explained that the team had rapidly yet carefully introduced clear practices for ensuring turn-taking and confidentiality for the new online drop-in youth work sessions. They then found that these were being taken up by the young people in their own online social groups.

We are getting very nice reports about those spaces feeling like an extension of the youth group spaces and being very supportive and young people giving each other very nice accolades and peer support within that. [youth work manager interview 2020]

Before the lockdown, workers had been very careful to maintain boundaries between their own and young people's online lives. As youth workers their role was to promote self-worth, facilitate the sharing of skills for privacy and to be alert to and responsive when safeguarding issues emerged. They felt they needed to respect professional boundaries and young people's privacy in such a way that involved avoiding mixing in both on and offline commercial LGBT+ spaces, for instance by avoiding personal Facebook and Instagram accounts.

The necessity to create shared digital spaces during the lockdown demanded that these boundaries be reworked. Rather than digital spaces simply existing through commercial platforms or communities of interest, these youth workers became adept at co-producing new digital spaces that were queer, informal, noncommercial and intergenerational using platforms such as Zoom and HangOuts. Drawing on their youth work practice they generated ground rules and thought through the affordances of the technology and reframed questions of access and privacy. To their surprise and delight the young people attending these groups took these practices into new spaces.

They have set up their own support mechanism, their own social spaces and they govern them in ways that do not feel at a high risk and in any sense they just have a sense of fun about them [..] the [our] ground rules have gone through into those and social spaces, but also the idea of you do some chatting and then you do an activity and then you'll say farewell to each other so that that kind of routine and ritual as models as well. lyouth work manager interview 2020]

This story helps us understand how far perceived generational gaps in expertise change how we experience digital technologies. It also shows that there is nothing fixed about this landscape, we are all often in the process of 'catching up' and remaking boundaries and there is something inevitable about getting it wrong, learning from our blunders and making new practices as a result. It also reveals the dynamic relationship between virtual and face to face spaces, that virtual spaces are not the same (a youthwork zoom room is very different to a dating app) and that safe intergenerational spaces can be generative and precious.

The research literature supports our findings about the unique role of community organisations in supporting LGBT+ youth. They provide spaces to openly explore identity, access LGBT+ specific resources, provide access to a sense of safety, what Bain and
Podmore (2020) characterise as "more-than safety" in the form of meaningful relationships (or attachment) with others. They provide spaces and structures for peer learning, intergenerational mentoring, social support and validation or positive identity development through mirroring, empathy, positive representations, self-defining, and a language for recognising oppression. Asked what was needed in order to support young people's queer world-making, a specialist provider explains:

Money! Funding that is not project based, that allows the youth workers to have a living wage, and comfortably live, so there is not a high staff turnover. [...] Organisations like ours, are picking up the failings of the state. Schools, social care doesn't have enough time to deal with this and the depletion of youth services in local authorities, and generally spaces for youth have disappeared. So charities and people with good intentions have to pick that up, and it all costs money. [specialist youthwork and health provider consultation 2021]

Voluntary service providers, which initiate provision from the bottom-up, often do so without substantial resources. In alignment with studies such as Paceley et al., (2015, 2016), they highlight how provision might need to differ by location, with non-metropolitan areas necessitating different strategies by LGBT+ youth for whom privacy, invisibility, and boundary work may be more essential than in cities. Continuing our research through lockdown enabled us to document how the move to online services during this period extended access, especially for young people in rural and suburban areas.

Digital youth work has had a massive update, hugely increasing our numbers, particularly those 
from rural areas, who had previously been unable to travel into our youth groups. There was a dip in summer, but we always do, even face to face. We have definitely made our service more accessible online and that is something we will continue to do regardless of what happens.[..]

Another barrier of face to face is using public transport. Although we have a bursary for travel, they have to pay upfront and claim the money back. But if you have an internet connection and a phone, which most young people do, either at home or in school, they are able to access our services.[..]

We've only run a couple of youth events, but we had a much greater geographical spread represented, and young people seemed to find the digital context easier from an accessibility perspective in a number of ways (particularly around anxiety) [specialist youth work and health providers consultation group, 2021]

\section{Conclusions}

This chapter is framed in terms of celebration in order to draw attention to the creative but everyday experiences that arguably constitute the majority of online experience including for LGBT+ youth, rather than focusing primarily on the negative ones. By acknowledging and attending to the practices of 'queer worldmaking' on the part of young people, we recognise how LGBT+ identities are relational and affirmative and operate in diverse spaces that link generations, allies, support work, education and community. This chapter points to the value of asset-based approaches to young people which recognise them as skilled, innovative and resilient navigators of the online. Their knowledge and experience of identity-building online goes beyond 'dating' to include queer 'digital socialities', developed through selfies, memes, gifs, emojis, comics, and avatars.
This approach challenges adults working with young people not to overlook the capacities of young people. The research literature and the evidence gathered in this project suggest that LGBT+ youth have much to teach. Finally, celebration serves as a counter to the dominant discourses of risk, vulnerability and sexualisation that are most commonly associated with youth, especially with LGBT+ youth, online. Issues of equity or safety should not be disregarded, but the vitality of the online needs more attention than it is commonly accorded. 


\section{CHAPTER 4: \\ EQUITY}

In this chapter we review the evidence collected in the study through the lens of equity. We have chosen to adopt the terminology of equity rather than equality, as it enables a discussion of structural and systemic issues, including policies and practices that shape economic and social institutions and produce injustice. A concern with equity encourages a focus on the resources needed to rectify inequality, rather than allocating resources equally to all recipients. The digital landscape, we argue, is a space where diversity appears to be recognised and catered for, but it can be underpinned by an inaccessible and surveillant architecture which negatively impacts LGBT+ young people. We look at public institutional spaces such as schools which are a matter of concern for LGBT+ young people, as the struggle for equity is experienced acutely here. We also consider the potential for relationships and sexuality education to be inclusive of diverse gender and sexual identities as well as considering whether and how schools can be safe spaces for LGBT+ young people, and why this is important. The chapter ends with a discussion of family, home and community and how access to emotional resources in these spheres can enable the capacity to thrive in the face of injustice.

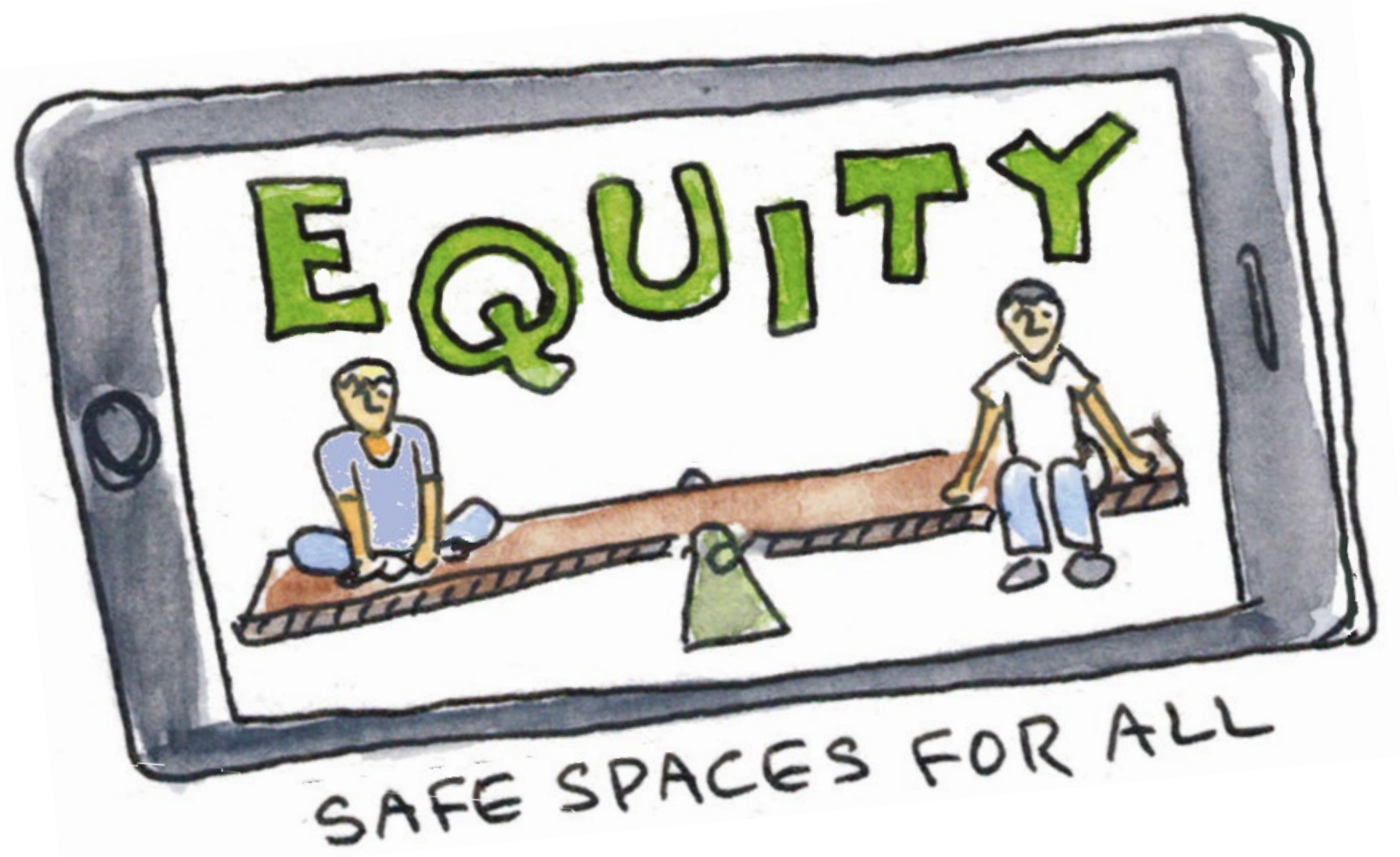




\section{The spaces for equity}

Many of the contributors to this research spoke in terms of wanting equality for LGBT+ youth. Curiously, few demands were addressed explicitly to digital platforms, possibly suggesting how successfully companies have absolved themselves of broader social responsibilities and liabilities. They looked particularly to schools to deliver on this equality. Since 2019, schools in England have been encouraged to deliver relationships and sex education (RSE) - a shift away from the more genitally focused 'sex and relationships education' that preceded it. This shift signalled a commitment to an education approach that was inclusive and accessible for everyone. Our participants suggested that this was still a work in progress, debating whether specifically targeted approaches were appropriate and suggesting that they could be divisive. They also wanted the spaces of home and family to be nurturing and safe ones.

Early internet research often expressed a view of the web as a comparatively free and safe space. It was anticipated that the internet would enable the creation of shared spaces within which ideas and experiences could be expressed that might be marginalized elsewhere. The idea of 'counterpublics' captures this vision (Fraser 1990, Warner 2002). This utopian view has been tempered in recent years. A more nuanced view explores how counterpublics are shaped by the affordances of different platforms (e.g. Reninger 2014). Concerns have grown as to the problem of harmful counterpublics (for example promoting hate speech, radicalisation and toxic masculinities, e.g. Ging \& Eugenia 2019). Increasing critical attention has focused on the competing imperatives of companies, advertisers, policymakers and users, and on the extractive character of commercial online spaces and the harvesting of data through user profiles (eg Wight 2014). What has been termed 'platform' or 'surveillance' capitalism (Srnicek 2017, Zuboff, 2019) is designed to extract robust and verifiable user data for monetization and therefore profit. Rather than disrupting the status quo, social media may also play a role in shaping and limiting user creativity (Keller 2019) and reinforcing normative conceptions such as gender binaries. For example, sites like Facebook have been criticised for broadcasting activities to networks without explicit consent, others for reauthorising binary gender by the demand for state-validated identity in the design of sign-up functions (Boyd 2011, 2014; DuGuay 2016). Filters in 'safety' software may block queer youth access to LGBT+ sites and platforms. These drivers carry specific challenges for LGBT+ youth who may need to work harder to keep parts of their identities and lives separate. Debate and conflict around LGBT+ rights 'are increasingly materialising within technical functions of internet governance and architecture rather than at the surface level of content' (DeNardis and Hackl, 2016). Research by Jenzen and Karl (2014) suggests that LGBT+ youth can be critical of digital culture, including being aware of how the algorithms underpinning search engines structure access to material.

\section{Different and the same}

A concern with equity focuses attention on the resources needed to rectify inequality, rather than only on equal resource allocation. Young people in the study were emphatic that, despite diversity under the LGBT+ umbrella, the shared experience of marginalisation (within heteronormative cultures) brought them together. The urban 16-25 youth group explore this as a political question that highlights common oppression across differences:

\section{Burt: For the majority the} experience of being $L G B T+$ is generally going to be the same because you don't fit into like the cis heteronormative view of the world if you like ... but there is differences between the way that some groups will experience things. Like. trans people will experience things differently to cis people and $\mathrm{Ace}^{13}$ people will experience things differently to not-Ace people ... and like each group is going to have something slightly different that it's combating but ultimately everybody is combating against heteronormativity, just slightly different.

A younger group at the same service similarly identify oppression as a shared experience:

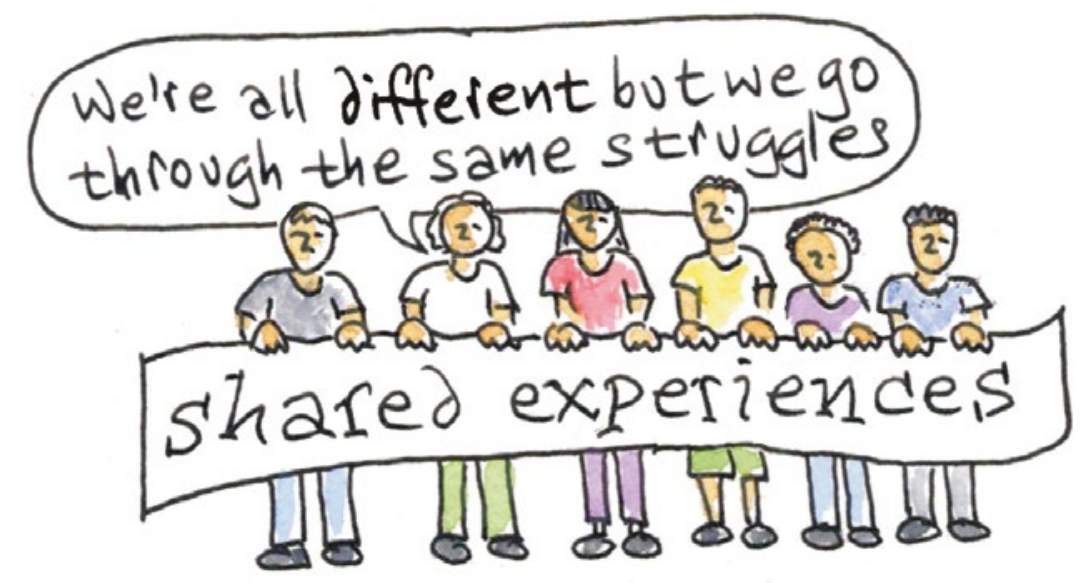


Maisie: I think, even though obviously different LGBT groups are different, I think actually we probably face a lot of the same kind of struggles.

Moca: Although they are a little bit different but we all have like similar struggles. Because people who are outside of the community sometimes don't understand how difficult their words are like when someone uses the... am I allowed to use slurs?... like when people say "faggot" or "tranny" they don't really know the implications of that so like people in the community, they all understand what it means. Clock: And it's horrible.

Moca: So, they're all connected ... They're more connected than you think. [urban, under 16 reference group 2020]

This sense of solidarity coexisted with an appreciation of how online networks could cater for and reflect diversity, enabling the formation of smaller communities of interest around particular identities but also around shared interests (such as fandom, anime and gaming communities). In contrast, institutional spaces such as school curricula appeared to them to reflect an older sexual politics and a preoccupation with heterosexual risk. A key issue raised by young people in the 2017 survey and focus groups was the inability of school Sex and Relationships Education (SRE)14 to engage with the spectrum of sexual and gender identities within which they understood themselves as being located.

Luna: There was, like, either heterosexual and some people were homosexual, [....] Apparently there's a line that you're either, either end [...] where, where does, like, trans people and bisexuals come on here, and pansexuals? It's just apparently, er, heterosexual and homosexual, there's nothing in between apparently, which people can't get their heads around. And it doesn't help that, like, er,

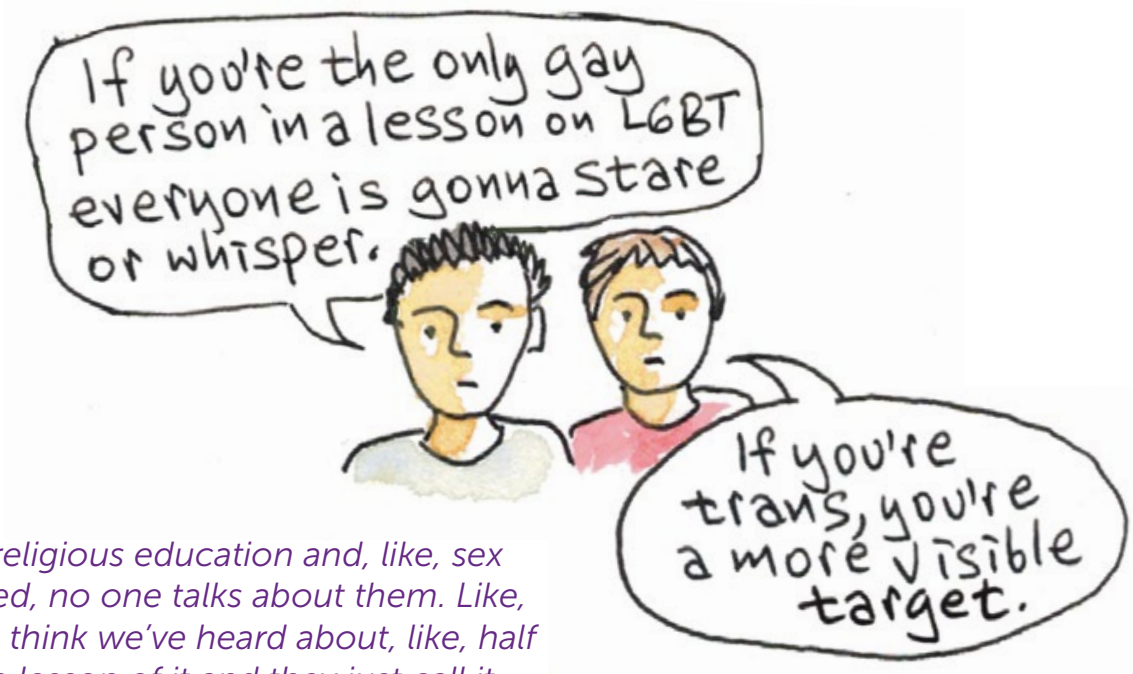

a lesson of it and they just call it, "The gays," they don't even go into pansexual, transgender, they don't say anything about LGBT+... [2017 focus group]

In this research the focus of young people's criticism was firmly on the shared public spaces of schooling, perhaps reflecting their assumptions that questions of citizenship and inclusion are officially on the agenda in these spaces. A key finding from the 2017 survey was that only $20 \%$ of all respondents reported a good experience of RSE that helped them to develop skills for relationships, dropping to only $14 \%$ of LGBT+ respondents. Focusing on LGBT+ respondents we find that $28 \%$ judged their education on positive/equal relationships to be 'not great', compared to only $15 \%$ of straight young people surveyed. Almost $30 \%$ of LGBT+ didn't receive any support in this area at all.

In open questions and in focus groups conducted as part of the 2017 study LGBT+ young people demanded more diverse and inclusive RSE. This included the need to get beyond the heteronormative, penis-in-vagina (PIV) model of sex that currently dominates the curriculum and a more serious engagement with questions of power, abuse and healthy relationships. The following responses from the open question provide a sense of what LGBT+ young people are looking for from educators. The 2017 survey question asked 'What if anything could staff in schools be doing to support and empower young people to have good relationships online and offline?':

Don't skirt around the issue - make sure the technicalities of healthy and unhealthy relationships and how to prepare yourself for dangers are very clear, make use of assemblies and lessons to discuss these topics much more so, and have in-school support systems in place to help those in unhealthy or abusive relationships and for those at risk. [aged 16, female, White British/English, bisexual]

Be completely honest and talk not only about basics or worstcase scenarios but everything in between as well! [aged 18, female, mixed/multiple ethnic groups, bisexual]

LGBT awareness. Teach us about gay sex health risks and how the power dynamic in same sex relationships can be a struggle. [Aged 17, male, White British/ English, gay]

Stop being so heteronormative! [aged 14, female, White British/ English, bisexual]

Teach us about gay sex not just about straight sex. [aged 17, female, mixed/multiple ethnic groups, bisexual] 
Be aware of the LGBT community. These relationships come with a whole new power dynamic and sexual risks that staff are currently completely unaware of. [aged 17, male, White British/English, gay]

These 2017 survey responses also suggest the need for a skills-based online safety curriculum that is relevant to the lives of LGBT+ young people and takes into consideration the affordances of digital technologies, both positive and negative:

\section{I think staff in schools have to} be really careful about how they approach the subject of the internet with students as often student take it, within my own experience, as them being annoyingly persistent and outdated. [aged 15, female, White British/English, bisexual]

Give $12+$ year olds information about sex - not just the biological side of it, also pleasure, protection and consent - in terms of online, give information about sending naked pictures that will be helpful and not scaremongering. [aged 16, female, White British/ English, bisexual]

Don't be anti-online; accept that it'll happen and talk more about safe ways to do it, rather than just saying, "No, it's not a good idea, never do it" etc.

[aged 14, female, White British/ English, bisexual]

The idea that school RSE is out of date brings together a critique of an old-fashioned binary of sexual politics (straight and gay) and a failure to engage sensitively with contemporary digital cultures. New conversations with young people in 2020 confirmed and deepened this critique. The young people in the reference groups were all attending youth clubs that provided support and education around personal relationships, and so might be better supported than the majority of young people responding to the 2017 survey. As part of the reference group discussions we invited young people to debate the proposition that Schools are not a safe space to do LGBT+ inclusive sex and relationships education. Young people emphatically asserted that schools should be safe spaces and that making this happen is a priority and a legal right. In Moca's words: 'you're at school so like at school, what, six and a half hours five days a week it should absolutely be a safe space.' Max Power lays out a vision in which inclusive RSE could be the means to making schools in general safer and more inclusive:

They are not a safe place yet. The only reason that they are not a safe place is because the school itself is not embracing the idea of teaching gay sex education and gay relationships education to its students ... students are more liberal and more kind than we give them credit for. They're more intelligent than we give them credit for. If schools actually buckled down and taught students LGBT+ information that would make their school itself more inclusive it will absolutely be safe enough to do so, without a doubt. [urban, 16-25 reference group 2020]

A couple of young people identified the religious affiliation of their schools as a potential barrier to inclusion, with staff citing fears around parental views as an obstacle. However, the more common view was that it is the prejudices of other students that make schools unsafe spaces for LGBT+ youth. For example, Sophie shares her view that:

I'm saying schools aren't a safe place for it. I've had we've all had some pretty horrific stuff said and done in school ... I suppose when you are trans it's a lot more visible, you're a lot more of a visible target... [In] schools everyone is still young everyone is still learning everyone is still making mistakes not everyone is ready to hear about that and while I think should be done, I don't think it's a safe space [rural, mixed age reference group 2020]

Moca explains the challenge in making schools a truly inclusive space, in a contribution that reflects on individual relationships between students but also highlights the role of broader school cultures in creating - or failing to create - safety:

Schools absolutely should be a safe space for anyone, like absolutely anyone, like your race, your gender, your sexuality. Absolutely anything. But like with the kids and their upbringing you don't know how they're going to be. It's like people in my school they are not necessarily like intentionally transphobic, [...] they misgender me and like I'm "can you not do that" and they're like "why" and I'm like "cause I'm not a girl" - and they're like oh okay and like if they accidentally do it on accident, I don't mind, but if they purposefully do it like the one kid at my school then like I'm obviously gonna retaliate. [urban, under 16 reference group 2020]

\section{A space within a space}

Retaliation from the margins can be emotionally exhausting as LGBT+ youth attempt to bridge the gap between community spaces (which recognise gender fluidity and a continuum of sexual expression) and heteronormative cultures (Gabb et al. 2020). Attempts from the LGBT+ community to intervene in the formal culture of the school are important and are welcomed. For example Maisie talks about her surprise at how ignorant school peers were when an LGBT+ group provided a session in Personal, Social, Health and Economic Education: 'they [fellow students] 
didn't know anything ... what asexual or pansexual or biphobia homophobia [were]. They were asking me every single one'.

The possibility of creating safe spaces within the context of the school was discussed in some of the groups, with young people talking about clubs and drop-in spaces created for and by LGBT+ youth, allies and those questioning their identity. In the urban under 16 group, Lamp talks about an 'LGBT allies group' that 'not many people know about'. In the 16-25 group, Gregg (who is a teacher) talks about the safety of their classroom 'where I do challenge things', but 'in the corridor' safety is less assured. In the rural location, Joker and Harley Quinn also debate the value of 'secret and confidential spaces' that involve some kind of invitation or referral by teachers, to more open spaces that can include where you can go 'to support a friend and... to learn about LGBT+ and the whole... shabam'.

Embracing diverse sexualities and gender expressions within the curriculum was seen as an essential part of building a more inclusive society and challenging hate crime. But at the same time, young people spoke about feeling exposed, with responsibility placed on them for educating others. Here Joker, Harley and Lauren share their experience:

Joker: It's technically hard in my school because I'm technically the only out kid in the school because of parents ... my friends aren't confident enough

Harley Quinn: Thing is if you're that open person everybody knows your sexuality and they start talking about it, eyes on you and then you're just there like "no" and then you feel really uncomfortable even though it's stuff that you need to learn and other people need to learn

Lauren: Yeah so if you are the

only gay person in your class and you had some sort of you know education about LGBT people you'd be scared that everyone is just gonna stare at you because they know that you're the only gay one or they're going to judge you or whisper to their mates about what they think so. [rural, mixed age reference group 2020]

Other young people talked about how a group of LGBT+ students could support each other. Maisie explains:

There is more than 1000 people in my school so obviously that's a lot of kids that are LGBT, which is really nice actually. Because yeah I have a friend in my form who is Bi - and I remember when we came out to each other it was really nice it was so casual. [urban, under 16 reference group 2020]

Michael also talks about a class where 'quite a few of us are LGBT', yet having to deal with 'insensitive and invasive' questions during a lesson on transgender. John elaborates on his point, explaining the relationship between an inclusive curriculum and a safer space for LGBT+ students.

I'm not sure. I think that some of the students would have, you know, as Michael was saying maybe be a bit invasive about it or not very nice about it, as it, cause, it doesn't really apply to them they might not realise that actually it might be really insensitive to other people, and you know I think, I don't think people would be as respectful as they could be. So, if we had education where we learned about everything, so we learned about heterosexual couples and pansexual couples and stuff like that, then I think that people would then grow up to be more - they would then grow up to be more liberal with their views if they might not be before. So, I think the education has a lot to answer for. [small city, mixed age reference group 2020]
The tension between accessing useful education for themselves and becoming an education for others, runs through the young people's accounts from both the 2017 and 2020 stands of research. Important gaps in RSE were identified such as risks associated with girl on girl 'there's not really any information on how to do it safely and that they mainly just touch on you know man and man sex not female female or anything like that just they do straight gay but not girl on girl' (Harley Quinn). Schools are not the only source of information and young people access multiple resources, including digital media. They understand that 'LGBT+ accounts and stuff' might be a better way to find out more about things that might be more personal, specialist, or part of the intellectual and cultural resources of a community.

\section{The challenges of inclusion}

\begin{abstract}
A focus on equity highlights the relationship between heteronormativity, bi/homo/ transphobia, discrimination and risk. The academic literature encourages a shift away from the individualized focus of concepts of 'bullying' towards one that more thoroughly accounts for 'how socio-economic processes, and diversities (in terms of intersecting class, race, cultural aspects) inform girls' and boys' gendered relations and conflicts within and beyond the school gates' (Ringrose and Renold, 2010: 592).
\end{abstract}

As the accounts of young people suggest, there is a strong critique of school-based RSE which is part of a wider conversation about how LGBT+ young people are able to move between the different spaces of their lives, which include the playground as well as the classroom, the street, the home and community and commercial spaces, online and offline. Young people recognise that as a group, LGBT+ youth have different needs, 
yet the demand that schools should be safe and accountable spaces is articulated in a clear and shared manner.

Consultations with practitioners, both teachers and specialist youth work and health providers, suggest that there is a need for work in this area, yet exactly what that work should be is far from clear. Existing curricula and their delivery need to be more inclusive. These points were made by teachers in a consultation group in response to the question: how might we make relationships, sexuality education (RSE) more inclusive?

The way we refer is really important. So, for example, "people with" uterus, vagina, it's very subtle, but it's therefore not excluding somebody who may or may not feel that they are or are not female, anyone. Same with condoms, talking about internal and external condoms rather than the femidom. It's very subtle, but I think it can help within the session for people to be able to relate to it, wherever they are coming from. The more you do that the more it becomes the norm. Little changes can make a big difference to those in the room. [teacher consultation, 2021]

If LGBT+ young people feel that RSHE is not inclusive, or helpful, and if that's their feeling, and that's because of general subtle wording, they will put the walls up potentially at the beginning of the session so you have lost them already. The aim is to educate and inform so they are safe, ultimately, so if it's about changing slight wording, it's helpful to be aware. [teacher consultation 2021]

Yet there is also a strong message about the dangers of stigmatising young people. In our 2020 survey of practitioners $67 \%$ of respondents strongly agreed or agreed there is a need for more resources for LGBT+ young people on the topic of online safety. However, $21 \%$ of respondents strongly disagreed or disagreed of whom $89 \%$ (18 out of 22 ) were engaged in delivering education on online safety. Open comments from this part of the survey elaborate some of the tensions involved in a commitment to inclusion:

- I don't know if we should treat young people in the LGBT+ world differently from other young people with difference. I think we should just ensure that all resources that are produced are all encompassing and do point out that we should be kind, respectful and thoughtful to others regardless of their difference.

- We don't have LGBT+ specific materials as we have a range of young people in our lessons and cannot single people out. Our young people are also still often questioning therefore we don't want to pigeonhole them, there is much more fluidity around sexuality now.

- Internet safety covers everyone across all genders. I don't see the point of designing something specific. Young people are clever enough to realise and understand what bits of information they need for their needs/issues.

- I think that the issues children face are wide ranging, and focusing on LGBT to such an extent risks additional stigmatising.

- We all too often sexualise youth work when we equally have a role in protecting their childhood. On one we have a duty to make young people aware of the dangers out there, on the other hand we must recognise that young people have many and varied challenges growing up and sexuality/gender is only one part of it. [practitioner survey 2020]

Among those who agreed on the need for new and better materials, there were also expressed concerns that an inclusive curriculum depends on an inclusive environment which in turn needs to be addressed through the wider environment and behaviour policies.

I put 'agree' as opposed for 'strongly agree' as I think there needs to be more sex and relationship inclusive resources across the board. I also think the issues mentioned above like, inclusive education and reducing bullying, harassment and stigma will reduce the risks for $L G B T+$ young people online rather than just addressing this through the production of online resource which may or may not be used. [practitioner survey 2020]

While some stuff targeted at LGBT+ is a good idea, more important is that general materials should be inclusive, and that presenters/teachers/trainers/ leaders should take the effort to be inclusive of different sexualities and gender identities in their discussion, as well as taking the effort to question/break stereotypical assumptions about the differences in vulnerabilities between boys and girls - as well as including different abilities. [practitioner survey 2020]

Again, we return to the tension between the diverse nature of those gathered under the LGBT+ umbrella, yet shared experiences of oppression arising from heteronormativity. In the consultation group with specialist providers of LGBT+ youth support, concerns were raised about ongoing government-level commitments to challenge trans, bi and homophobia in schools through antibullying and other policies concerning harassment and abuse.

Funding from GEO ${ }^{15}$ has stopped for LGBT+ anti-bullying in schools, and also taken away healthcare for trans young people. We don't 
know what the impact will be. It is not driven by the young people who require services. [specialist youth work and health provider consultation 2021]

In LGBT+ community spaces young people are able to become ordinary and diverse with many different needs, rather than being sexualised as a group because of their identifications. The ideal is for schools to become spaces where this can also be recognised. RSE is part of this process, but care needs to be taken that LGBT+ identities do not become the lens through which young people are defined or understood as a problem.

Young people are exactly the same as their straight peers except for their sexual and gender identity. It's only part of who they are and it doesn't define them. [specialist youthwork and health providers consultation 2021]

\section{Home is where the heart is?}

Equity continues to be a struggle for LGBT+ youth faced with the everyday realties of sexism, heteronormativity and harassment. Despite the efforts of individual staff members, evidence from the wider research literature confirms that gender-diverse youth experience discrimination within the school environment from the curriculum, space, peers and teachers (BowerBrown et al., 2021; Bragg et al., 2018). 'Home' too can be unsafe and unsupportive, even abusive, demanding additional emotional labour from young people that itself takes a toll on mental health (Gabb et al., 2020), especially under lockdown conditions (Fish et al., 2020; Hanckel and Chandra 2021). Privacy (online or off) can be particularly challenging for those in poverty (Vickery 2015). But it can also be a resource that defends young people against the damage caused by inequities in the world beyond. We end this section by thinking about questions of equity in relation to domestic and family life, reminding readers that the context collapse brought about by digital technologies (Boyd 2011) disrupts notions of public and private spaces and in doing so raises new questions about the appropriate spaces for making and claiming equity.

An important finding from the 2017 Digital Romance study was that supportive relationships with parents could be a protective factor for online harm. For example, the ability to communicate with parents/ carers may be important when the 'dramas' (Marwick \& Boyd 2014) of online sociality explode. Yet home life was also identified by practitioners surveyed in 2020 as an area of vulnerability for LGBT+ young people, especially young people from families where diverse gender and sexual identities were taboo. The worry was that 'children are afraid to come out to parents and therefore not able to discuss relationships online, so lack of parental support/oversight' [survey comment 2021].

We were interested to explore relationships with parents and carers in more detail in the reference groups with young people. One of the statements used in four groups as a provocation for discussion was 'I can tell my family/carers and friends about how I use digital media', and it resulted in an interesting and diverse conversation. The first, perhaps most significant, finding is the wide range in experience reported by participants regarding how supportive parents are and thus how open they can be. Accounts ranged from complete estrangement and secrecy to complete openness and acceptance. The urban youth centre that hosted two of the reference groups works closely with parents, and many of the young people in this setting report very positive relationships with families. For example, Maisie (in the under 16 group) talks about her parents being 'very chilled and very liberal'. In the 16-25 group Jimmery describes herself as 'very lucky', 'I don't feel I have to hide that side of myself'. While not everyone in this setting were 'out' with parents (one participant for example describes family as having 'broken down'), the young people share mostly optimistic accounts of family life that included Max Power's rueful admission 'My dad suggested that I get into dating apps which is probably the saddest moment in my life'. It is worth noting that even where relationships are good, young people present themselves as fortunate - Moca explains the norm for LGBT+ young people is 'being kicked out of their homes, and like being abused because you know, they're gay'.

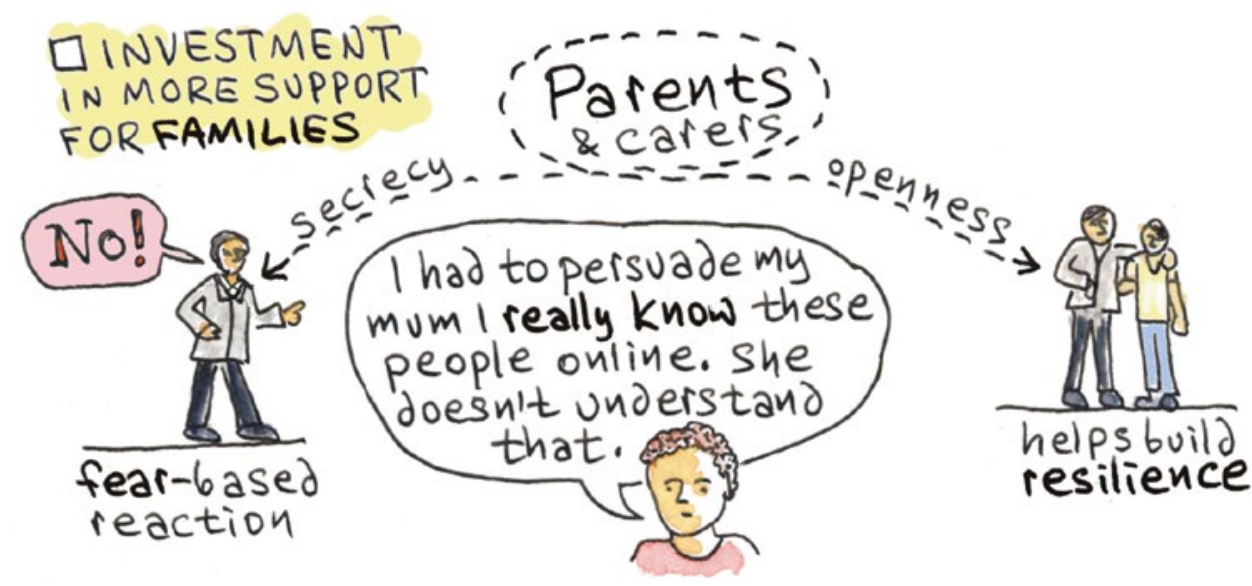


In the more rural settings the picture was more mixed, and the discussion gave rise to some painful feelings in the group, captured here by Sophie:

OK, so I think it's great that you're close to your family, I think that's a really sweet story I feel like the world needs more of your family, so kudos to you- But I think for a lot of us it's not that easy. Like when I came out to my parents, they burst into tears [rural, mixed age reference group 2020]

In the same group, Joker describes their family as 'unsocialised', explaining 'we keep ourselves to ourselves, we don't really talk'. In the small city group, Lauren describes her mum as 'not really a supportive person', explaining 'I wouldn't feel comfortable talking about anything'. Michael explains he is 'a private person, so I don't tell my parents much about my life' while adding that 'if I needed to go to them about something I think they would listen'.

The accounts of the younger people tend to express frustration with protective parents. So, for example, Clock complains 'I don't like it when my parents find out who I am contacting because it's my friend.. my private conversations.. they go through my messages to see if I'm being sensible or safe'. Lamp agrees, complaining about their mum wanting to look at their phone 'whenever I'm on my phone and laugh at a meme'. The term 'overbearing' is used by both Lamp and Moca who are exasperated by parental concern over meeting friends they have met online. Lamp complains about 'making a big deal out of it saying I should be careful and not reveal anything'. Moca also berates her mum for making 'a massive deal'. The idea that it is possible to make friends and 'know' people online is something that parents are seen to struggle to understand.
These questions of boundaries and privacy are discussed further in Chapter 5 (Safety). In the context of a discussion of equity we note that the making of boundaries with parents is an important part of the work of the teenage years and it is not surprising that younger participants were preoccupied with breaches of privacy. The 'default publicness' that is designed into many social media platforms to facilitate profit-making may be especially difficult for LGBT+ young people for whom such 'publicness' may be dangerous (Cho, 2018). At the same time, phones may feel as if they are extensions of the self (Albury \& Byron, 2016) characterise them as 'affective technologies': both touched intimately and held close to the user's body, and invested with their users' desires and emotions. These tensions between public and privateness are illustrated by Moca in the under 16 urban group. Moca wants to be close to their parents but does not want them following their Instagram account:

Because it's very close to me... I'm going to be very honest, most teenagers nowadays they are very personal with their social media, because that's sometimes the only place they have. Having an adult look through your phone without consent is a very big breach of privacy that parents should not do.

Older participants were able to reflect back on these kinds of parental anxieties and to see them in retrospect as expressions of care, even when warnings were ignored by the young person. John's self-description is as 'an open person, I'm not ashamed of anything. If my mum wants to see my phone, I would give it to her straight away'. He explains:

When I was younger, there were times when I wanted to meet people I didn't know and she had been umming and ahhing annoyingly about it. [..] she would only let me do that if it was with someone who I had met before, who I knew, no one like an adult [small city mixed aged reference group 2020]

Jimmery cringes at the thought of parents seeing videos of nights out on their social media: 'they've seen me so drunk that I couldn't remember who I was... they've looked after me in those situations'. Yet Jimmery also has boundaries 'I wouldn't talk to my family about what I do on dating apps because that's a bit odd ... a bit too far open.' [urban, 16-25 reference group 2020]

In the same group, Alan, who selfdescribes as 'not completely out', has a carefully curated Instagram that his parents follow, explaining 'there's a lot of my online presence but also like my life that I wouldn't share with my family'. In a similar vein, Gregg describes their Facebook page (followed by sister, mother and cousin) as 'carefully orchestrated to present something people would interact with. I don't think anyone in my family knows I have functioning genitalia.'

A vision of equity within the home and in relation to family may seem strange from within a tradition of equalities work that focuses primarily on public institutions, access and level playing fields. Yet it is important that we factor it into our thinking about LGBT+ youth including inequities in access to support and protection.

Increasingly, specialist providers are working directly with parents and carers as a key strategy for building resilience among young people over the long term. In consultations with parents as part of this research we became aware of a huge area of unmet need for information, discussion and peer support. Parents look to schools and other formal institutions as vital spaces for reliable information and positive values. As one parent said: 
We do pin quite a lot of hope on proper teaching of RSE, and if more opportunities for LGBT+ young people to see themselves represented. So much in the media that is so false, and sensationalist, so if through teaching and information there could be more normal stories that would be good. [parents consultation 2021]

Another parent who runs a peer support network comments:

We get contacted by parents to ask if it's ok for their young people to have a sexual relationship, and so we have to say the age of consent is 16. We have to remind them that this is not different for LGBT+ young people. The issues are the same, but because the support is lacking already, they need more support now. [parents consultation 2021]

\section{Conclusions}

In 2003 the law was changed equalising the age of consent for all sexualities. In 2010 the Equalities Act made 'sexual orientation' and 'gender reassignment' protected characteristics, on the basis of which discrimination is unlawful. Yet in 2021 these policies still need to be turned into lived equity. In this chapter we have considered some of the resources needed to rectify inequity, such as inclusive RSE, safer school cultures, attention to how online spaces breach rights for the sake of profit, home as a safe space.

A key part of this debate is understanding how heteronormativity generates risk for gender and sexually diverse young people. Inclusive practice becomes safer when heteronormativity is noticed and addressed. Without these underpinnings, targeted work has the potential to be stigmatizing. Evidence from specialist providers and from young people themselves show that it is possible to create spaces in which LGBT+ young people can be ordinary and diverse. Yet schools are not alone as public spaces. This chapter encourages an understanding of spaces as networked (as they are for young people) with the potential for positive and hybrid 'counter-publics' to be linked across home, community, school and online, commercial spaces: and to address demands to all of them accordingly.

\footnotetext{
${ }^{13}$ Ace is an umbrella term used to describe a variation in levels of romantic and/or sexual attraction, including a lack of attraction. Ace people may describe themselves using one or more of a wide variety of terms, including, but not limited to, asexual, aromantic, demis and grey-As. https://www.stonewall.org.uk/help-advice/fags-and-glossary/glossary-terms. A glossary of terminology is included in appendix 2

${ }^{14}$ In England, what had since 2000 been called SRE was, in 2019, reframed as RSE, with guidance noting the requirement for schools to comply with the Equality Act 2010 , explaining 'we expect all pupils to be taught LGBT content at a timely point as part of this area of the curriculum' (DfE 2019).

${ }^{15}$ Gender Equality Office
} 


\section{CHAPTER 5: \\ SAFETY}

In this chapter we focus attention on questions of safety and risk in relation to digital intimacies for LGBT+ youth. It is important that this discussion is contextualised by an understanding of the benefits of digital culture and broader questions of equity. Yet it is also important that the (albeit uneven) risk for LGBT+ youth is recognised. The chapter begins by acknowledging the potential for online harm to LGBT+ young people, before going on to explore how living with risk is an 'ordinary' part of growing up for LGBT+ youth. We then focus on how and from whom young people learn safety strategies before ending with a discussion of the role of educators.

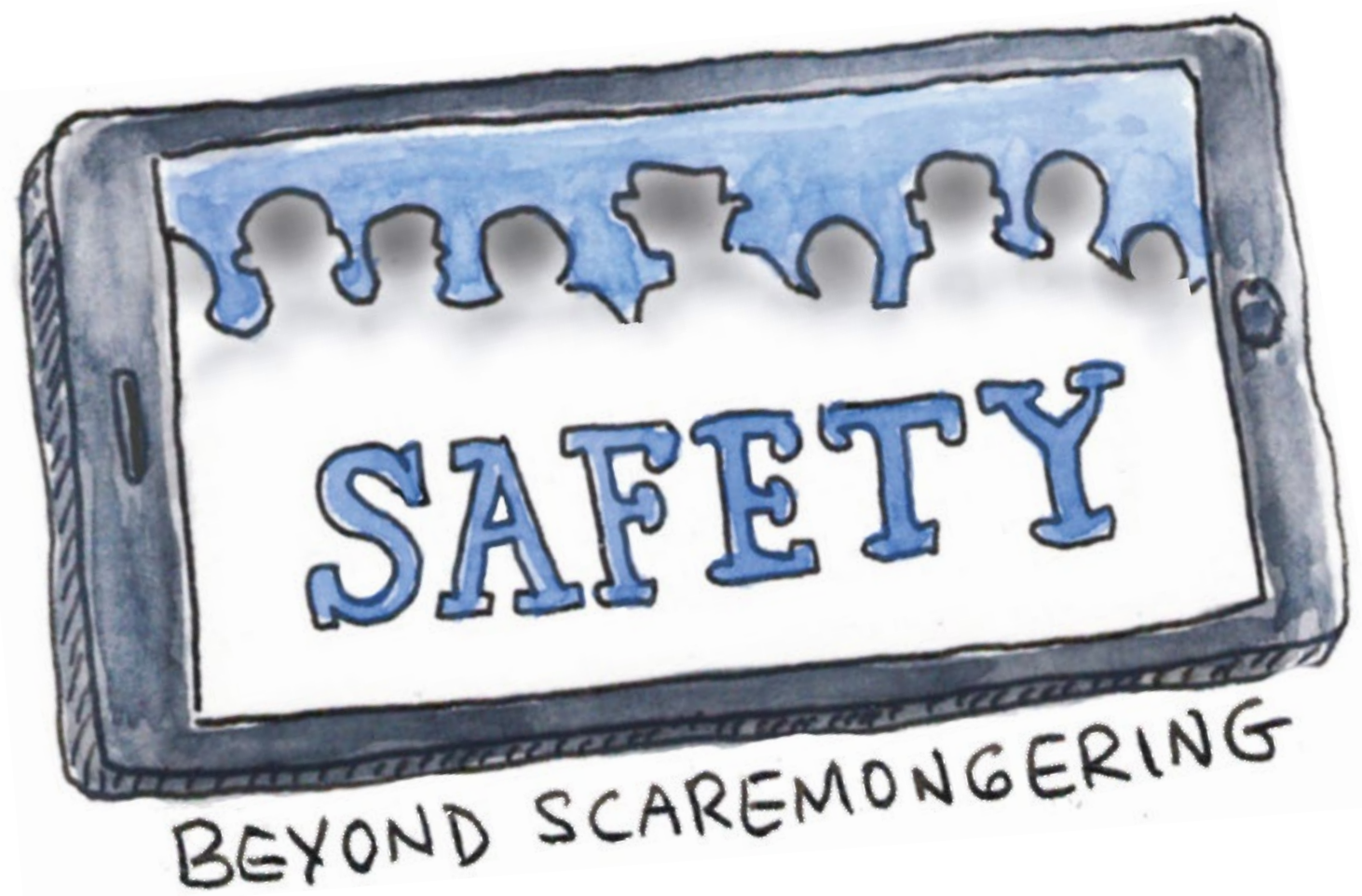




\section{Recognising risk}

Across this research, from the different stakeholders involved, there was a concern with ensuring the safety of LGBT+ young people in their use of digital media and in the process of growing up as confident and secure young people able to make positive relationships. There was also recognition of the real effects of heteronormativity, meaning that LGBT+ young people may encounter more or different risk than their cis-straight peers, faced with a range of challenges including isolation from community, bullying and harrassment from peers, and rejection by family. The search for acceptance, belonging and intimacy are likely to be bound up together resulting in a greater reliance on digital methods for meeting others and self-discovery.

Young people were adamant that online sociality could be a space for self-making and community (see Chapter 2 Celebration), yet they were also frank about the perils of life online. A key message from the LGBT+ community involved in this research (young people and adults alike) was that risk is uneven, and that although the effects of heteronormativity produce a powerful binary between cis-het and 'others', in practice the extent to which individuals experience risk is shaped by specific factors. Young people with special educational needs $\&$ disabilities (SEND) may face specific (and diverse challenges) and young people without the protective resources of a supportive family and/or community may have less resilience in the face of the kinds of inequities that arise from heteronormativity (see Chapter 4 Equity). Economic risk is also vital, amplified by lockdown, with some young people unable to afford to get online, and others drawn into risky money-making activities.

\section{Creeps and bullies}

During this research we heard a number of personal stories from young people, from parents and from professionals that confirmed that there are genuine risks of exploitation and harm facing LGBT+ young people. For example, one of the parents in a consultation group shared a story of discovering by chance her son's contact with much older men and the unsafe advice he has received online.

\section{I picked up the iPad that I thought} he wasn't using and he had all these males that were 20 years, 50 years plus that had been messaging him... he was then in this massive new world, where people were saying, it's ok if you are gay, as people can go to the Travelodge and pay to have sex. [Parent consultation group, 2021]

In one group a young woman recalls an experience of attempted grooming:

One person was being very weird towards me and then I realised that they wanted like - that they were hoping that, keep in mind I was nine years old and they were being very flirtatious towards me, and they had a profile picture I think they were on a role playing account and they had a profile picture of one of my favourite characters. And I just I kind of went along with it until they started asking me very, very personal questions and then to the point of like what's your password like they thought that I'd like I mean I kind of was like lured in - but then at least I knew not to get people my password I learned that at Roblox ${ }^{11}$. [urban, under 16 reference group 2020]

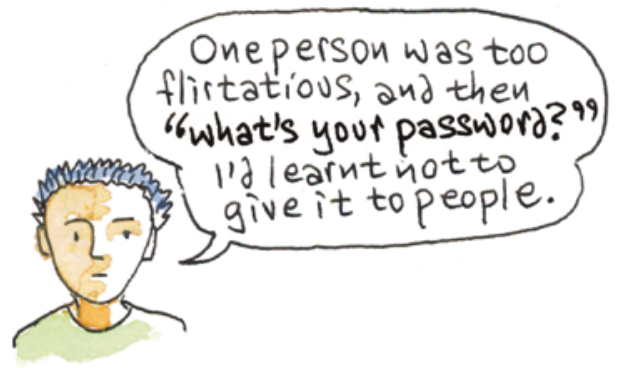

Young people consulted in this research talked about 'creeps' as being part of the online landscape, and something that it was necessary to understand and to learn how to manage:

Sophie: I think LGBT+ people are vulnerable because of prejudice and discrimination and not particularly just ... you know creeps, some people in the LGBT+ community are creeps, so you know it's not necessarily that as just more discrimination... I mean throughout centuries we've been you know murdered or put on display just because we like the same sex or we like both or you know we're a woman instead of a man you know. [rural, mixed age reference group, 2020]

Joker: Well it's like, all our Mums have told us at some point that there's some nasty man on the internet out to get us and while that's a teeny bit exaggerated, not every internet friend is a creep from somewhere ... however these people still do exist and some of them have preferences that are disturbing to say the least and vulgar to put it lightly. But I feel like it's important to kind of keep these people far far far away from normal society especially, if they like to target young people such as ourselves. [rural, mixed age reference group, 2020]

The findings of the 2017 Digital Romance survey reveal how LGBT+ youth may be differently positioned in relation to risk compared to their cis gendered and heterosexual peers, for example, through being more likely to meet up with someone first met online.

- More non-binary gender young people $(55 \%)$ had met someone online who they started seeing compared to cis-gender girls (37\%) (38\% of boys reported this). - More bisexual (42\%) than straight (36\%) young people had done so. 
- More non-binary gender young people had asked someone out (on or offline) $(64 \%)$ than cisgender girls(34\%); (56\% of cisgender boys reported this).

- More gay (58\%) and bisexual (52\%) young people had asked someone out online compared to those who were heterosexual (38\%). These differences are all statistically significant (McGeeney \& Hanson 2017: 17).

As a group, trans young people in particular seemed to face risks in both online and face to face settings. So, for example, in the 2017 survey only $33 \%$ of those identifying as transgender felt that face-to-face was the best way for someone to flirt with them (significantly lower than cis-gender young people: 63\%). Transgender survey participants reported feeling more confident and sexy flirting online than off, and more nervous and uncomfortable offline than on ${ }^{12}$. Young people who identified as transgender were significantly also more likely than non-trans young people to have experienced judgments on their appearance of all kinds: offline and online, positive and negative ${ }^{13}$.

The uneven and intersectional character of risk was commented on by young people:

\section{I think everyone experiences things different based on who you are and all the factors that people are based on - disability, sexuality, your race, all of the above and stuff. But I think definitely that being like a white person or a Black person, trans, cis, whatever. I think would definitely make an impact, like I think the number of Black trans women murdered in America is depressingly high and it's not the same as white trans women. There's... because you have to ... it's not just the homophobia, there's the racism, it's layer on layer of discrimination. It's not just one type. I'm aware that I tick a number of the boxes}

in terms of representation and discrimination and stuff. So, it's something that I'm aware of. [Nora interview, 2021]

And by specialist providers:

It's really important to frame research and report, that $L G B T+$ young people are not only LGBT+, they are also able bodied people, disabled, come from a wide variety of ethnic backgrounds, neuro diversity, family situations and so it's almost impossible to categorise a whole group just by looking at LGBT+ people. You have to separate that out as all intersectional. How young white straight men get along in society is wildly different from how a trans young person of colour gets along. [specialist youthwork and health consultation group, 2021]

Practitioners also drew attention to the particular situations of young people with special educational needs, noting for example that 'young people questioning sexuality with learning difficulties (including autism/Aspergers) face additional challenge and can become very fixated on online relationships' [practitioner survey 2020]. This message was echoed by a parent who explained that her autistic son missed the nuanced messages of school RSE and safety because ' $\mathrm{He}$ is very literal, and you need to tell him something clearly'.

The idea that young people might turn first to the internet as a space to ask questions about their sexuality and gender identity was a concern for those supporting them. In many cases, parents only begin educating themselves once something has happened to alert them to a problem. As one parent explained:

Online is good, but be careful what you search for, need safe organisations, so you don't come across something shocking. I didn't have the vocabulary to know what to search for. The stuff I looked at in the beginning was not something I would think important now. [parents consultation, 2021]

The unfiltered nature of the internet caused concern among practitioners, not only because of the risk of young people accessing adult content but also discovering sites that promote conversion therapy or unsafe sexual practices.

- They can access anything without any censorship, they can look at any aspect of sexuality before they have developed an understanding of what it means.

- I worry about where they might be looking for support/what might be influencing them. Kids with gender dysphoria worry me as I know there are some harmful methods that some kids in desperation will turn to and I worry if this might be promoted anywhere online.

- Distress as a result of anti-trans/ anti-gay/deprogramming content online, including anonymous abuse but also websites, some of which look quite official. Parents being targeted by anti-trans/ anti-gay groups and groomed to protest against schools/ withdraw support from children/ target teachers etc. Online misinformation and intolerant language causing anxiety, polarised and frightening debates online especially on Twitter and YouTube. [practitioner survey 2020]

As the material gathered in this section suggests, there are both generalised risks facing LGBT+ youth associated with the effects of social exclusion (for example bullying and harassment) as well as quite specific risks which may be associated with intersecting inequalities, specific sexual identities, communities and associated platforms (for example the risks associated with $\mathrm{CHEM}^{16}$ sex subcultures). So, for example, in our consultation with specialist providers of LGBT+ sexual health advice we were 
also alerted to 'other forms of risk-taking behaviour such as drinking and smoking so in general good health messages are not effectively reaching them' and the normalisation of 'certain sexual practices that are unsafe'.

It is worth noting that public health research appears to be most familiar to practitioners, but this literature has a perhaps inbuilt orientation to risk. It tends to focus on 16 or $18+$, as well as men who have sex with (cis gender) men (MSM), a group identified as in most need of interventions due to (for example) HIV transmission rates and compounding needs, sometimes referred to as a 'syndemic' (Bonell et al., 2020) related to substance misuse, poor mental health, negative self-image, social isolation to which youth is particularly vulnerable (Prock and Kennedy, 2017). Within this literature less attention is given to groups considered low-risk (such as lesbians), to positive or healthy rather than problematic behaviours or relationships, to the romantic rather than the sexual, to mental as well as sexual health (AgnewBrune et al., 2019).

\section{Risk is normal}

It is important to acknowledge the reality of potential harm for LGBT+ youth (online and off) and the considerable concern about protecting young people from harm shared by parents/carers, practitioners and young people themselves. It is also important to look at risk as part of everyday life, as a factor that is constantly managed and negotiated, and to think through the place of risk in the processes of growing up LGBT+. While their use of digital technology may make LGBT+ youth more vulnerable in some respects, it may also make them more 'savvy' than straight and cis young people. The wider research literature emphasises young people's acquired skills in negotiating safety, intimacies and pleasure in such spaces. For example, the work of Byron and Hunt (2017) stresses that gender and sexuality diverse young people's everyday practices of learning, teaching, knowing and sharing information often happen at a distance from formal settings, or within these but beyond the instruction or supervision of adults. Keller's work (2019) shows activist young women negotiating the 'vernaculars' and affordances of different social media platforms. These points are echoed by Lauren in our research:

Lauren: 'I think that most of them are quite mature and kind of know their way around social media. I imagine that they've been on social media quite a lot in their life obviously because they find it easier to meet people there. So, I think they might already know the risks of the dangers that are already out there, and I don't think they'll need as much help as people who aren't on social media as much, because they have been educated or they've educated themselves' [small city, mixed age reference group, 2020]

John also articulates how experience of oppression might improve understanding of risk:

\section{I think that LGBT+ people suffer} more oppression in general in their lives, so they're more likely to know because they're more likely to be bullied and oppressed because they then have learned the hard way, more likely, than straight people who haven't been oppressed for their sexuality. [small city, mixed age reference group, 2020]

The accounts of young people gathered in this research suggest that cautionary tales of 'near misses' are circulated between young people, who are in turn sensitised to risk by these stories of how they might be targeted:
Michael: 'you just kind of see it and like "oh that could be me" and like "okay l'll just be careful" you know it's not gonna necessarily stop me from doing anything but I'm aware of things like that happening you know and I'll be cautious about it' [small city reference group, 2020]

Albury and Byron (2016) argue that a certain level of distrust is a default mechanism for assessing and managing risk. In order to engage competently in contextual risk-assessment processes it is first necessary to recognise the dangers of exploitation and deception. As the following extract from one group discussions suggest, young people in the qualitative part of this study exhibited this necessary distrust:

Sam: anyone can set up a very convincing profile if they want to [...] Jimmery: you can never know the kind of person that someone is and everything you can do to try to keep yourself safe, there will always be risks.

Alan: You're not gonna pick like a photo that looks really really old and ... they're probably not that age anymore or a photo that's just their abs like you know some of the basic ones. [urban, 16-25 reference group 2020]

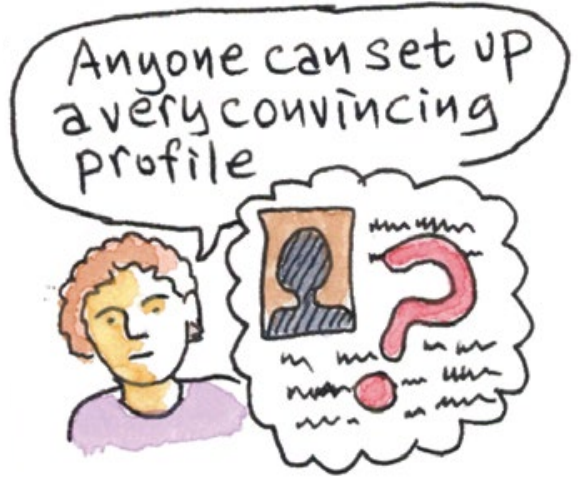

We accessed these young people through youth services. These services generally provide access to reliable health and wellbeing education and referrals, making it possible that our participants are better informed and supported than others who may be more 
isolated from expert peers and adults. Yet as Albury and Byron (2016) also argue in their research on 18+ LGBT+ youth in Australia, risk should be seen as an intrinsic aspect of online engagement, not as something that can be eliminated, or even avoided, but something that must be recognised and managed to mitigate the worst harms. These conversations about risk are nuanced, with different risks associated with specific online platforms and a specific understanding of each is needed to stay safe. For example, Lauren explains:

I think that all apps are as dangerous as each other because there's always one thing on each app that you should be careful. Like, so Snapchat obviously, don't send anything inappropriate because it can stay there forever, they could screenshot it and spread it. Facebook, really, don't talk to people who don't have any mutual friends or have just found you out of nowhere, and then Instagram, you've got people that are trying to follow you then you have any idea who they are and always liking your pictures always saying how gorgeous you are even though they don't know who you are. [small city mixed age reference group, 2020]

Anthony suggests that learning how to manage 'creeps' on Grindr is part of learning to be a gay man: 'unfortunately, like, a lot of things, you do find the one or two occasional creeps, but if you can get past them, then you'll probably eventually find a nice guy.' [interview, 2017]

Sophie's comments capture the bitter-sweet combination of pride, vulnerability and determination that shape the landscape of digital intimacies for LGBT+ youth:

\begin{tabular}{ll}
\hline Responses in order of frequency to question in & No. of \\
practitioner survey: 'in relation to groups of LGBT+ & mentions \\
young people you work with, what are the key & \\
challenges and concerns you have about online safety?' &
\end{tabular}

Grooming/exploitation/meeting strangers - worries 22 especially around YP changing age to access unpermitted sites, power and age dynamics in LGBT+ communities

\begin{tabular}{ll}
\hline Lack of community, not 'out' to friends and family & 19 \\
\hline (Cyber)Bullying & 19 \\
\hline Lack of access to (reliable) information & 8 \\
\hline What's online, accessibility of 'disturbing' content & 7 \\
\hline Dating apps/online dating & 5 \\
\hline Lack of LGBT+ specific information (in schools) - increases & 4 \\
\hline likelihood of finding inaccurate, damaging sources online & 4 \\
\hline Yexting sharing personal details & 4 \\
\hline Catfishing - (though this ties in with grooming/ & 3 \\
\hline Parents/family not fully understanding risks of the online & 3 \\
\hline \begin{tabular}{l} 
Lack of online role models/representation \\
\hline
\end{tabular}
\end{tabular}

There are risks and problems everywhere - But I think like he said it's hard to meet people online, it's a lot easier especially for me who despised myself with every inch of my being... but online is a lot easier to kind of like, you know, meet someone and chat with them and kind of like hit it off and do some things you shouldn't, but, what the hell - enjoy when you are young. So don't give me that look, you've done it too ... yeah, it's a lot easier too when you are -- what if you aren't comfortable with your body you just go online and be yourself like on Snapchat you can have an avatar which you can customize to look however you want so that was great for me [rural, mixed age reference group, 2020]

\section{From scaremongering to safeguarding}

A common feature of public and policy discourse about young people's online behaviours is to frame the online 'world' as inherently dangerous for young people. For LGBT+ young people, especially, risk dominates the research agenda (Jones, 2013) with the public health literature especially constructing LGBT+ young people in terms of risk (Byron, 2015) and vulnerability due to negative self-image and social isolation (Prock and Kennedy, 2017). In this context the focus of policy-makers and in school settings is primarily on 'e-safety': increasing awareness of risks such as grooming, catfishing ${ }^{17}$ and image abuse; and teaching practical and sometimes technical fixes to stay safe. 
The online survey of practitioners conducted as part of this research shows a high level of engagement with different online safety resources and concern about how to ensure that safety messages reach $L G B T+$ young people.

Practitioners were alert to the heightened dangers faced by LGBT+ youth, with 'grooming and exploitation' being the most common concerns reported in the practice survey (followed by 'lack of community' and 'cyber bullying'). Online dating apps were a worry for some of the practitioners surveyed, especially in terms of the potential for misrepresentation in terms of age, but also the potential for bullying and harassment as a result of catfishing. Comments from the 2020 practice survey included a range of concerns. These include the way that young people questioning their identity may turn first to the internet and to dating sites such as Grindr, 'meeting adults' and 'creating profiles reflecting an adult age when they are children'. Responses acknowledged why young people may do this - 'our young people do not get many opportunities to meet other LGBT+ young people from outwith the area', and 'the young people we work with seem to be very needy for affection and attention'. The factors contribute to a perception of vulnerability, both in terms of encountering adult sexual attention but also the mischievous attention of peers who may 'pretend' to be who they are not. As one practitioner comments: 'at least one of our young people recently had an online friendship/relationship with another young man who turned out to not be real (it was other young people "catfishing")'.

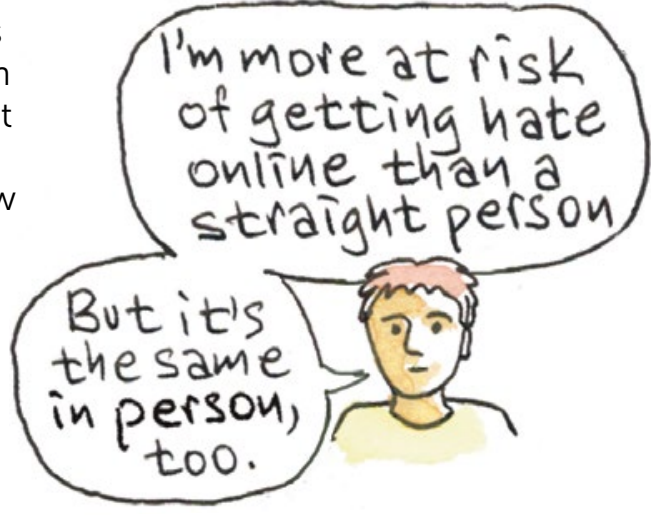

An important message from this research is that an emphasis on risk may alienate the very people that it aims to protect. The following contributions by LGBT+ youth respondents to a question in the 2017 survey about how to improve online safety education capture this sense of resistance to negative messages:

Teach them better e-safety rather than just 'stranger danger, the internet is scary' so young people feel unable to ask for help - victim blaming is so prevalent so they need to be more accepting. [2017 survey aged 16, female, White British/English, bisexual]

Don't make it sound really scary - staff often said we should never talk to people online. Showing good examples of different relationships (especially healthy same-sex relationships). [2017 survey, aged 21, female, White British/English, bisexual]

These questions were raised in consultations with young people. Asked by the facilitator whether the risks are so great that young LGBT+ people should avoid going online, Michael describes this as a form of 'victim blaming'18 which would penalise those who are reliant on those spaces for so many aspects of their lives (small city, mixed age reference group, 2020). His point is expanded by Nora who explains how an exclusive focus on the online space deflects attention from how safety is also a question of equity and inclusion:
Like, as a queer person I'm more at risk of getting queer hate online than, you know, a straight person is. And that makes me less safe online. I don't think that changes whether online or in person. Like I know in the city, my girlfriend and I would be walking out and suddenly she'd drop my hand and I don't know why but I look ahead and see a group of guys that probably would do nothing to us, might not even be homophobic, probably won't be but it like ... the worry. We don't know it's instilled in us, that fear of that group of guys, and being gay, and needing to stop for a minute to act straight, act cis, act normal and just kind of conform to society's idea of the 'standard people' so we won't get hate for it. Yeah. I have to or I don't feel safe otherwise. [Nora, interview, 2021]

The perceived safety of handholding is a sensitive barometer of the complex interactions that are captured by terms such as 'inclusion' and 'safety ${ }^{19}$ '. Some of the accounts shared by young people in this research show how important it is to balance messages about risk with positive accounts of the potential for healthy relationships. For example, Max describes herself as:

Stupidly paranoid there is no way anyone can trick me out of my house. I am so - I've been trying to like build myself up to getting into like a dating app for like well over a year now ... and I got one, uploaded one photo, and then never looked at it again because I'm so paranoid, I'm just so paranoid of the idea of something bad happening. [...] I literally, I downloaded Bumble of all things that ... I never touched it because I was so scared of it, it's frightening stuff'. [urban, 16-25 reference group] 


\section{Learning safety}

As explored in Chapter 4 (Equity) there is a lack of consensus around the value of targeted work on e-safety for LGBT+ youth. Although needs may be quite specific there is a danger of sexualising and stigmatising a particular group of young people. Not only should resources be inclusive, but strategies for combatting heteronormativity could transform the formal and informal curriculum. The accounts of young people consulted in this research suggest that they benefited from generic online safety received at school. For example, Maisie enthused:

In primary schools I think we learned about this website I think it was called cyber cafe 9 [...] and I think that definitely helped a lot [..] I remember I used to play this online game called Club Penguin and I was obsessed with it they had this [...] online safety test on there, which I think is really good because a lot of young kids played the game and they might not know like Internet safety and stuff like that. [urban, under 16 reference group, 2020]

Alan's description of his own online safety strategies suggests that it is an ongoing life-project that grows alongside your digital footprint. What distinguishes those who are endangered and those who aren't may involve luck as well as skills and knowledge:

I kind of grew up on the internet... and I do think like I theoretically know a lot of the stuff to do with safety. But I feel like sometimes it can be one of those things that like you kind of know but you don't necessarily get on top of all of it. It's like backing up your data, like you kind of know that you should do it. And sometimes you do do it, but at the back of your mind you probably haven't done it in a while. There's a few things with that where I just think, I think I was lucky. Especially like when I was 14 and probably like told people too much about my life online and then like kind of reined it in. And then it comes up on Facebook memories and I delete a bunch of stuff. But I do think you can never be too educated about it. [..] nothing really terrible has ever happened to me to do with meeting someone [...] and I do think part of that is luck. [urban, 16-25 reference group 2020]

Sometimes bad things do happen, and these, in turn, can be important learning experiences. John describes an incident that he made sense of by confiding in supportive adults in his life:

I mean, yeah, I've had like much older men try to add me, try to talk to me and try to get me to send naked pictures and stuff like that. And it happened to me, people who talked to me who have seemed - who have acted weird ... I can't really explain it, but it just made me feel really like unsafe. It just made me feel something's not right.[...] I've talked to youth workers about it a lot and I've had sessions on being safe online before. And I guess I've just learnt from the experience, like, I've talked to my parents about it. My parents knew that that was happening and I spoke to them about it, being really scared about it. And they, that's kind of giving me more understanding. So, now, yeah, I've just learned.. [small city, mixed age reference group, 2020]
Parents also recognised how trust needs to be built and renegotiated over time:

It's got to be consensual on both sides. The young person has to want to be helped. It's hard to get support and set boundaries, as they are pushing against everything.

It wasn't about the fact that he was gay, it was about safety. He did not know what was a safe situation and what was not.

It's not about setting a boundary/ restriction and saying no, as the forbidden thing is more appealing. Sometimes, boundaries are set because parents are scared. If information was shared and discussed so parents were not so scared, it could be discussed more broadly so young people knew better what the dangers were. [parents consultation group 2021]

Openness with parents is a process, with many young people needing to assert boundaries as part of developing their own lives and sense of self. It is not surprising then that managing privacy and the risk of being 'outed' by others online is a key concern for LGBT+ youth. In the wider research literature Hanckel et al., 2019 describe youth circumnavigating affordances, platform policies and norms to manage boundaries between what is 'for them' (family, work colleagues, friends) and 'not for them', in the process mitigating risks. The collapsing of boundaries between spaces

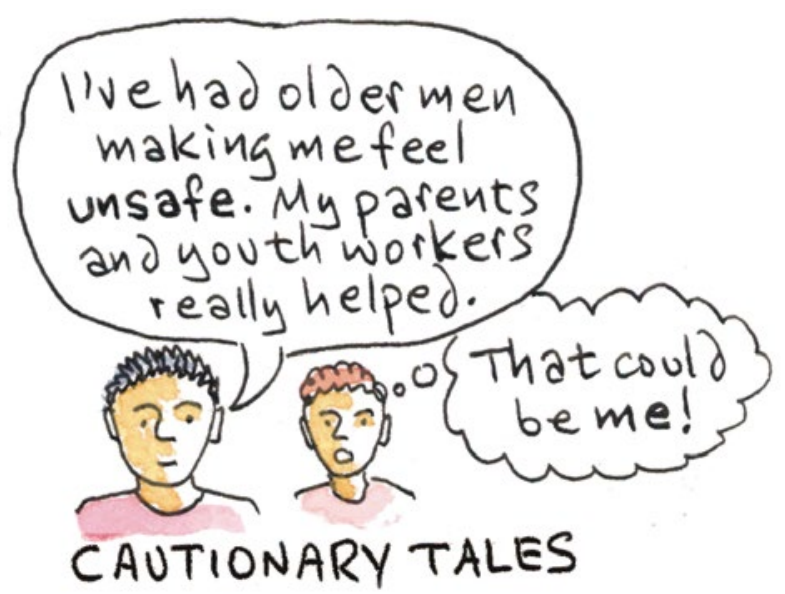


is an integral part of online engagement (Boyd, 2011) and young people may become adept in the digital literacies that allow them to maintain privacy - for example practices of concealing messages or information within other non-secret text or data. Duguay (2016) argues that these strategies depend on wider social norms such as 'civil inattention' by unintended audiences (such as parents). To read too closely can be interpreted as a form of 'stalking', and a violation of privacy. Her analysis refigures privacy as a dialogue that involves respect and negotiation on the part of adults, rather than solely responsibilising youth (Duguay, 2016). This balance is captured by Moca who appreciates parental support yet also warns parents about the dangers of intervening to 'take away the friends that help you cope with stuff' [urban, under 16 reference group]

Young people face very different situations regarding how safe or comfortable it may be for their sexual and/or gender identity to be known by others. Parents, family, neighbourhood, community, school/ college all constitute different audiences and it may take young people some time (if ever) to make their personal life public. Learning how to be safe in intimate relationships is a longterm project. Practitioners involved in this research acknowledged that LGBT+ young people may face greater risks than their cis gendered and heterosexual peers due to the inequities of, for instance, not feeling able to confide safely in others about whom they are meeting and what they are worried about:

\section{LGBT+ young people are more} vulnerable if they are facing issues of feeling not accepted at home or by their peers. ...for questioning kids who don't really have support at home / parents disapprove of them exploring in terms of their gender identity. I worry about where they might be looking for support / what might be influencing them.

Feelings of isolation young people feel when exploring their sexuality, conversation around exploration is not encouraged in society or education/ parenting. This means that young people may turn to less reliable more risky avenues to explore. Blackmail for children still not out to peers/parents, including for favours, sex, or as part of criminal exploitation. [practitioner survey, 2020]

\section{A model for understanding safety and risk for LGBT+ digital intimacies}

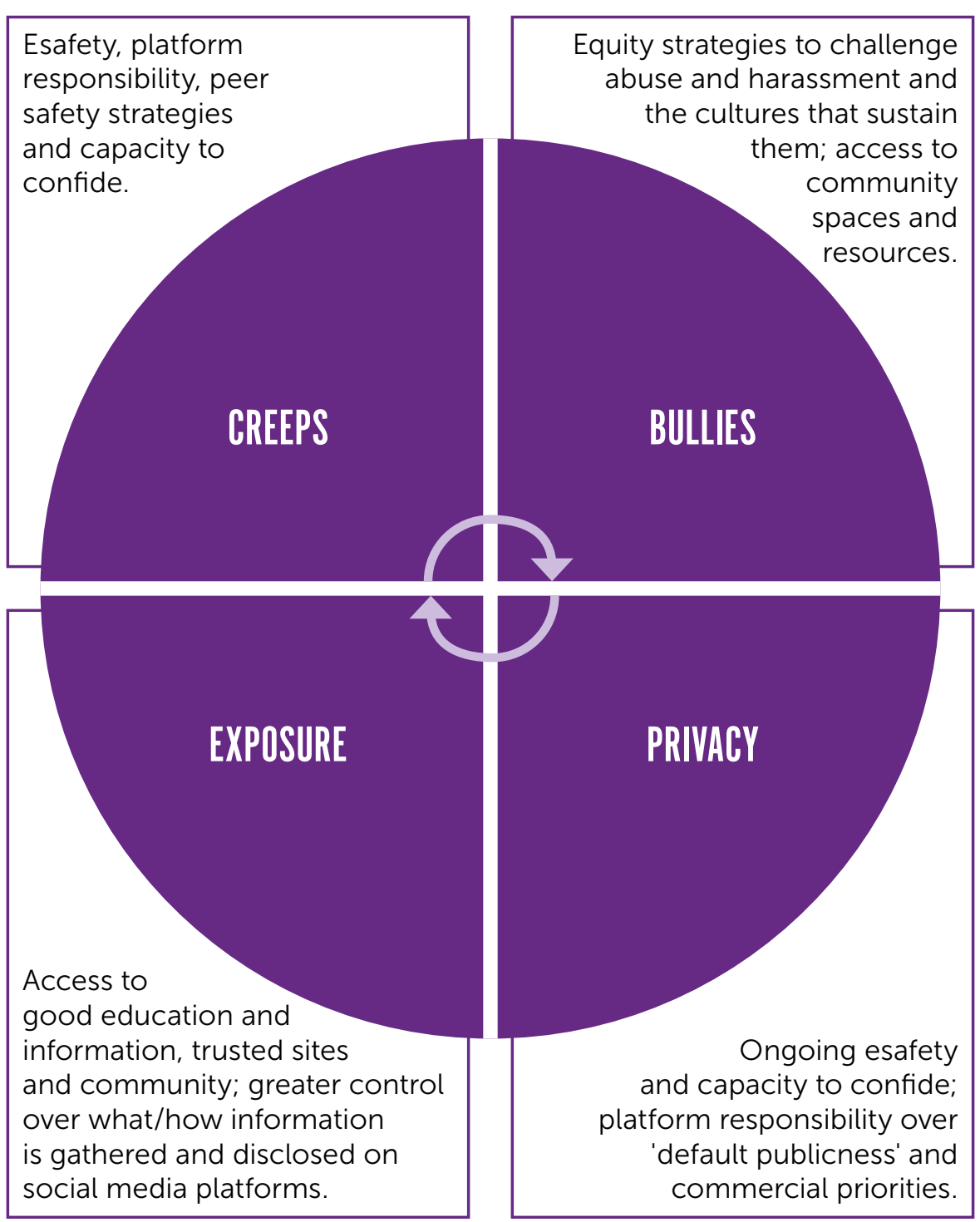

In drawing this chapter to a close it is possible to bring together the voices of young people, parents and practitioners to think about the relationship between safety and risk. From the perspective of young people, risk is made up of people (creeps and bullies) and transgressions (loss of privacy and unwanted exposure). We can think of factors that generate these risks, but also the factors that are protective and promote safety. Some of these are within the reach of young people and some have to be the responsibility of institutions, families and the wider polity. 


\section{Conclusions}

In this chapter we have focused attention on what it may take to ensure the safety of LGBT+ young people as they grow up and engage in friendships and romantic relationships in a culture where online and offline resources and practices are impossible to disentangle. We have deliberately positioned this as the last of the three findings chapters, in order to contextualise debates over online safety in a good understanding of the value of digital LGBT+ cultures and of the need to understand heteronormativity as a key factor in the potential risks LGBT+ young people may encounter.

We began by acknowledging the reality of risk and the potential for harm, drawing on the accounts of young people, parents and practitioners to identify key areas of concern including encounters with 'creeps' and bullies, the loss of privacy and the consequences that may lead from this, and exposure - to materials, sites and ideas that could be misleading and unsafe. These potential risks may be amplified for some by other inequalities, with SEND and family rejection featuring prominently in the accounts of practitioners. While we acknowledge these risks and the potential for harm as well as the concerns and fears of adults, in this chapter we also draw attention to the dangers of 'blaming the victim', curtailing young people's access to life-saving and life-enhancing resources and the capacity and luck to develop a life of their own. This involves thinking about and supporting safety, including the strategies that young people develop to share knowledge and help each other remain safe as well as how they respond to challenges when they happen.

The presence of supportive adults - be they parents/carers, youth workers or school staff - is a vital part of this picture, as is online safety and the promotion of a set of skills for reviewing and updating privacy and safety strategies. If LGBT+ young people encounter risks - especially those that are greater or different than their cisgendered and heterosexual peers - it is largely due to the effects of heteronormativity and there is a key role to be played in challenging trans, bi and homophobic bullying - which might be better thought of as a form of collective abuse or harassment - and ensuring that both RSE and online safety are genuinely inclusive. At the same time, access to community spaces such as LGBT+ youth clubs and projects is not only life saving for LGBT+ youth and their families, it also provides a space that goes beyond safety, a space where they can thrive.
The Covid-19 pandemic changed the landscape of risk for LGBT+ young people, most of whom spent long periods 'stuck at home' and accessing community spaces virtually. Our conversations with specialist youth workers during lockdown suggest that the character of risk has shifted, with more awareness of risk in the home (including LGBT+ youth as a focus for domestic violence) and the impact of economic risk on young people unable to access the usual sources of work available to the young in the services industries. A key concern also focuses on the post-pandemic world and whether the LGBT+ community infrastructure will still be intact - the network of safe and creative spaces that are so vital to the 'more-than- safety' message that we are conveying in this report.

\footnotetext{
${ }^{12}$ A total of 78 survey respondents identified as transgender, but only 56 answered these particular questions. Of this group, $39 \%$ of these said they would feel 'awkward' flirting face to face, while $50 \%$ said they would feel 'sexy' flirting online. Only $30 \%$ said they would feel confident flirting face to face which goes up to $40 \%$ when flirting via social media, apps and the internet.

${ }_{13}^{13}$ Negative criticism of appearance online had been experienced by $35 \%$ of those respondents identifying as transgender 'quite a few times' or 'a lot', compared to only $20 \%$ of nontrans. Offline, this had happened to $53 \%$ of trans young people and $29 \%$ of non-trans.

16 "Chemsex means using drugs as part of your sex life, and it's most common among gay and bi men. There are typically three specific 'chems' (drugs) involved. People say these drugs make them feel less inhibited and increase pleasure." Source: https://www.changegrowlive.org/advice-info/alcohol-drugs/chemsex-drugs

drugs make them feel less inhibited and increase pleasure." Source: https://www.changegrowlive.org/advice-info/alcohol-drugs/chems

${ }^{18}$ Victim blaming occurs when the victim of a crime or any wrongful act is held entirely or partially at fault for the harm that befell them. https://en.wikipedia.org/wiki/Victim_blaming

${ }^{19}$ See https://www.essex.ac.uk/research-projects/holding-hands
} 


\title{
SUMMARY FINDINGS \\ AND AREAS FOR ACTION
}

\author{
In this final chapter we identify areas for action, \\ based on the findings presented, to ensure LGBT+ \\ young people are full, equal and safe participants in \\ online activity and communities, and benefit from \\ the full potential of digital technologies.
}

\section{Summary findings}

\section{Celebration}

- The many positive and creative experiences that LGBT+ young people have online should be acknowledged;

- Community and other spaces need to be understood as transcending an online/offline divide;

- For LGBT+ young people in particular, there is fluidity between friendships and romantic relationships;

- LGBT+ youth spaces deliver more than safety: within them, LGBT+ young people can be diverse and 'ordinary';

- Generational gaps in expertise (perceived and actual) structure how we experience and feel about information technologies;

- However, generation gaps can also be transcended: youth work professionals who rapidly and flexibly adapted their online practices under lockdown supported positive online relationship-building by the LGBT+ young people who accessed their provision;

- Safe intergenerational spaces can be generative and precious.
Equity

- Despite diversity under the LGBT+ umbrella, there are shared experiences of oppression and marginalisation within heteronormative cultures;

- Relationships and Sex Education (RSE) is seen to be out of date, both in terms of old-fashioned binary sexual politics (straight and gay) and a failure to engage constructively with contemporary digital cultures;

- Schools should be a safe space for everyone, but they are not so yet for many LGBT+ youth;

- LGBT+ young people struggle to access useful education for themselves and face becoming an education for others;

- Inclusive curriculum depends on an inclusive environment;

- The commercial imperatives of digital media platforms create specific inequities for marginalised young people;

- The home continues to be a space of inequality, with a wide range in experience reported by participants regarding how supportive parents /carers are and thus how open they can be.
Safety

- Risk is real: there are both generalised risks facing LGBT+ youth associated with the effects of social exclusion and heteronormativity as well as quite specific risks which may be associated with intersecting inequities (eg ethnicity, religion, disability, poverty and geography), specific sexual identities, communities and the design of platforms;

- Risk is a normal part of teenagehood and needs to be recognised and managed to mitigate the worst harms;

- An emphasis on risk in educational approaches may alienate the very people that it aims to protect;

- Messages about risk need to be balanced with positive accounts and representations of the potential for healthy relationships;

- Online safety is an ongoing lifeproject that grows alongside a digital footprint;

- There is an important protective role for supportive adults with whom trust is built and renegotiated over time. 


\section{Areas for action}

Inclusive Relationships and Sex(uality) Education (RSE) RSE needs to be inclusive of non cis-heterosexual identities. This is a change that involves language, images and information, and also values, methods and responsiveness. Inclusive RSE not only plays a part in engaging and responding to the questions of LGBT+ youth but also in revealing and questioning heteronormativities for all young people.

Schools need access to support, training and resources from expert organisations to give them the confidence and skills to ensure that delivery of the whole RSE curriculum is inclusive. They need to ensure that their curriculum and approach is informed by student voice and reflects the lived experience of all in the school community.

However, care needs to be taken not to place responsibility onto LGBT+ young people to educate others, nor to see LGBT+ young people primarily or exclusively through their sexuality.

The RSE curriculum alone cannot make schools inclusive and safe, it is a necessary (and enabling) but not sufficient condition for this. Whole school approaches need to be developed to make the classroom, the corridor and the playground safe; and to provide support for those young people whose gender or sexuality make them vulnerable at home.

\section{Inclusive online safety}

Online safety education needs to acknowledge and respond to diverse gender and sexual identities and their intersection with other differences which may increase risk, including special educational needs and disabilities, ethnicity, religion and poverty. Adaptable materials that avoid ambiguity and treat online safety as a skills-based life-long project would be welcomed. Scaremongering should be avoided, with emphasis on promoting safety practices that require revisiting and updating over time in line with age, stage and digital footprint. Young people's existing knowledge and strategies in this area should be recognised and drawn upon, but they cannot be assumed.

\section{Resourcing anti-harassment strategies}

Challenging the broader cultures that enable abuse and harassment - going beyond punitive and / or individualised approaches - is necessary for LGBT+ equity and essential work for schools and other online and offline spaces.

\section{Demanding more from} digital media providers Schools, youth services and other public bodies are frequently tasked with providing solutions to many issues of (youth) community, risk and safety, often without resource provision to help them do so. However, what is also needed is a nuanced conversation about the responsibility of digital media platforms and companies to increase safety for LGBT+ young people while maintaining the accessibility of their vital online spaces, and relevant content.

\section{Actively supporting parents and carers}

Investing in the education and support of families and carers of LGBT+ youth is an important strategy for supporting the resilience, wellbeing and safety of young people. Inclusive peer support for and between parents and carers, including support during times of crisis, is essential.

\section{Creating and networking} safe spaces

A relational approach that acknowledges and connects the safe spaces for LGBT+ youth has value. It is possible to create spaces within spaces, marked by visual insignia and linked to other online and offline spaces that are experienced as safe.

\section{Supporting youth community spaces}

Sustainable funding for LGBT+ youth work and youth spaces within the LGBT+ community is vital. Having access to such spaces (online and offline) plays an important part in ensuring the safety and wellbeing of LGBT+ youth. They model positive intergenerational relationships within the LGBT+ community and provide trusted and authoritative points of contact for parents/ families, schools, colleges and the wider youth service. Most importantly, they provide spaces where LGBT+ youth can be ordinary, diverse and included; a place where they can thrive.

\section{Supporting creativity/ world-making}

Young people are creative within online spaces and can be agents of change in their own right. Investing in creative digital projects for LGBT+ youth and youth workers not only acknowledges the positive aspects of digital culture in young people's lives but would better equip young people to understand and make their own digital content.

\section{Outreach and exchange}

There is an important role for projects to facilitate LGBT+ community groups and 'ambassadors' to work in schools, colleges, universities, public and commercial spaces, digital platforms, to promote understanding of the needs, concerns and safety of LGBT+ youth. 


\section{Acknowledgements}

A number of researchers contributed to this project including Dr Khaliden Alsaleh, Rosie Gahnstrom and Ester McGeeney. The research team would also like to thank Kelly Harris and Helen Corteen at Brook for running the stakeholder consultation groups, and Ryan Gingell (Allsorts) and Sally Carr (Proud Trust) for sharing their professional insights. Laura Hamzic and Lisa Hallgarten at Brook made vital contributions to the final report as did our two academic peer reviewers Professor Jacqui Gabb and Dr Laura Harvey and our young critical friend Lily Halliday Marrable. It truly has been a team effort. Illustrations by Michi Mathias and graphic design by Sarah Ferrari. Thank you. Finally, and most importantly we would like to thank the young people who shared their time, thoughts and ideas with us. We hope you like the report! 


\begin{tabular}{ll} 
Key term & Definition \\
\hline ACE, Asexual & $\begin{array}{l}\text { An adjective used to describe people who do not experience sexual } \\
\text { attraction. A person might also be aromantic, meaning they do not } \\
\text { experience romantic attraction. }\end{array}$ \\
\hline Binding & $\begin{array}{l}\text { Breast binding is the act of flattening breasts by the use of constrictive } \\
\text { materials. }\end{array}$ \\
\hline Bisexual & $\begin{array}{l}\text { A person who forms enduring physical, romantic and/or emotional } \\
\text { attractions to those of the same gender, or those of another gender, in } \\
\text { different ways to varying degrees over their lifetime. }\end{array}$ \\
\hline Catfishing & $\begin{array}{l}\text { Denotes the fabrication of online personas to lure others into a } \\
\text { fraudulent relationship. }\end{array}$ \\
\hline Cisgender/cis & A term used to describe people who are not transgender. \\
\hline Cyberbullying & A term used to describe people who are not transgender and who are \\
heterosexual.
\end{tabular}

Facebook A popular free social networking website that facilitates online interactions through its interface for users aged 13 and up. *Note - Facebook has received criticism over the effect that its 'real name' policy has had a negative effect on the lives of various members of the queer community.[1]

FaceTime A video telephoning software released in 2010 for Apple products.

Gay

An adjective used to describe people whose enduring physical, romantic, and/or emotional attraction are to people of the same sex. It has also been used to specifically describe men whose enduring physical, romantic, and/or emotional attraction is for other men.

\begin{tabular}{ll}
\hline Gender Identity & Is a person's internal and personal sense of their gender. \\
\hline Gender non-conforming & $\begin{array}{l}\text { Relating to a person whose gender expression is different from } \\
\text { conventional expectations of masculinity/femininity. Note, not all gender } \\
\text { non-conforming people identify as transgender, and vice versa. }\end{array}$ \\
\hline Grindr & $\begin{array}{l}\text { A geo-locative social networking and online dating application, geared } \\
\text { towards gay, bi and trans identifying men who have sex with men. }\end{array}$ \\
\hline Heteronormative & $\begin{array}{l}\text { Denotes a view that promotes heterosexuality as the normal or preferred } \\
\text { sexual orientation. }\end{array}$ \\
\hline Heterosexual & An adjective used to describe people whose enduring physical, romantic, \\
& and/or emotional attraction is to people of the opposite sex. Also, straight.
\end{tabular}


Key term

Instagram

\section{Definition}

A free online photo-sharing social network platform that was acquired by Facebook in 2012.

*Note - Instagram has recently received criticism over its 'shadow banning' of queer and plus-sized users due to perceived sexually suggestive imagery.[2]

Kik A mobile messaging application for the exchange of messages, photos and videos. A key feature is its emphasis on preserving its users anonymity, by allowing users the option to anonymously send messages, and to register without providing a telephone number.

\begin{tabular}{ll}
\hline Lesbian & $\begin{array}{l}\text { A woman whose enduring physical, romantic, and/or emotional } \\
\text { attraction is to other women. }\end{array}$ \\
\hline LGBT+ & Acronym for Lesbian, Gay, Bisexual Transgender, and others. \\
\hline $\begin{array}{l}\text { Non-binary and/or genderqueer } \\
\text { and/or ENBY }\end{array}$ & $\begin{array}{l}\text { Terms used to describe individuals who may experience their gender as } \\
\text { falling outside the categories of man or woman. They might experience it } \\
\text { as in between, beyond or different. }\end{array}$
\end{tabular}

Outing/Outed

The act of publicly declaring or revealing another person's sexual orientation or gender identity without their consent.

Pansexual

An adjective used to denote a person whose enduring physical, romantic and or/emotional attraction is not limited in relation to sex, gender or gender identity.

Queer

An adjective used to denote people whose sexual orientation is not exclusively heterosexual.

Has also been used to denote people whose gender expression is not cis. It was once understood as a pejorative term, and its use is not universally accepted.

Sexting The sending/receiving of sexually explicit messages, images, video via messaging platforms.

\begin{tabular}{ll}
\hline Snapchat & A free social networking app that enables users to send videos, pictures \\
& or messages to other Snapchat users that disappear once the recipient \\
reads/opens them.
\end{tabular}

TikTok

A Chinese video-sharing social networking application that lets users create and share videos up to 60 seconds long.

Tinder

A geo-locative social networking and online dating application. It allows users to anonymously swipe to like/dislike other profiles based on photos and a short bio. The app does not target a userbase on the basis of sexuality/gender - the profiles like/dislikes are based on the preferences the user sets up.

*Note - Tinder has received criticism from trans-activists over their treatment of trans identifying app users.[3]

Transgender An umbrella term used to describe people whose gender identity differs from the sex they were assigned at birth.

Twitter

A free social networking microblogging service that allows members to broadcast short posts, called tweets, that are limited to 280 characters.

Yubo
French social networking app designed to "make new friends" and create a sense of community. Geared towards teenagers and young adults aged 13 to 25 . Allows users to direct message, video chat and livestream with up to 10 friends. Users can swipe right on someone else's profile to 'like' them, and swipe left to pass on to see other people's profiles. 25 million users worldwide.

[1] https://www.facebook.com/chris.cox/posts/10101301777354543

[2] https://www.theguardian.com/commentisfree/2019/nov/08/instagram-shadow-bans-marginalised-communities-queer-plus-sized-bodies-sexually-suggestive

[3] https://www.bbc.co.uk/news/technology-50390028 


\section{Below is a copy of the survey as shared with professionals in February/March 2020.}

We want to hear about the resources you are using when working with young people on online safety and/or gender and sexuality, your views on the needs of LGBT+ young people and your suggestions on future resources.

CEOP is a command within the National Crime Agency and is the United Kingdom's dedicated law enforcement unit for combating child sexual abuse and exploitation.

Brook is the only dedicated sexual health and wellbeing charity for under 25s. For over 55 years Brook has been delivering clinical services, as well as relationships and sex education in schools and other settings.

The survey should take an estimated 10-15 minutes to complete.

Thank you for participating in this Brook-Child Exploitation and Online Protection Command (CEOP) survey.

\section{Privacy and Confidentiality}

Brook promises to keep your information safe and protect it from being lost, damaged or shared with anyone it shouldn't be. If you have any questions about the use of your data you can read our full privacy notice on our web-site www.brook.org.uk/ privacy, speak to a member of staff to ask for a paper copy or you can contact Brook's Data Protection Officer at dataprotection abrook.org.uk or you can write to us at Data Protection Officer, Brook, 81 London Road, Liverpool, L2 8JA
1. Do you currently deliver education directly to young people on online safety and / or gender and sexuality?

a. yes

b. no

\section{[IF YES TO FIRST QUESTION]}

\section{About you - Practitioner}
What is your role?
a) Teacher
b) Teaching assistant
c) Youth worker
d) Relationships and sex educator
e) Other

What organisation type do you currently work for?
a) Primary School
b) Secondary School
c) PRU
d) SEN school
e) Alternative education provider
f) FE College
g) HE Education
h) Other training provider

Which age groups do you currently work with? Under 7
a) 7
b) 8
c) 9
d) 10
e) 11
f) 12
g) 13
h) 14
i) 15
j) 16
k) Over 16 


\section{[IF NO TO FIRST QUESTION]}

Do you manage or commission others to deliver education to young people on online safety and/or gender and sexuality?

a) yes

b) no [IF NO TO THIS - SURVEY ENDS]

\section{[IF YES TO QUESTION ABOVE]}

\section{About you - Manager / Commissioner}

What organisation type do you currently work for?
a) Primary School
b) Secondary School
c) PRU
d) SEN school
e) Alternative education provider
f) FE College
g) HE Education
h) Other training provider

Which age groups do you currently work with?

Under 7

l) 7

m) 8

n) 9

o) 10

p) 11

q) 12

r) 13

s) 14

t) 15

u) 16

v) Over 16

[QUESTIONS BELOW APPEAR FOR BOTH GROUPS PRACTITIONER MANAGER/COMMISSIONER]

\section{Your views on the needs of LGBT+ young people}

In relation to groups of $L G B T+$ young people you work with, what are the key challenges and concerns you have about online safety? If relevant, please explain what you see as the most important issues for different age groups.

[FREE TEXT]

What specific groups of LGBT+ young people do you think have particular needs?
a) LGB young people
b) Trans young people
c) young people with autism
d) young people with special education needs (SEND)
e) faith groups
f) young people in care
g) other (please specify)

Please let us know how much you agree with the following statement

There is a need for more educational resources for $L G B T+$ young people on the topic of online safety
a) Strongly agree
b) disagree
c) neutral
d) agree
e) strongly agree

Can you please tell us the reason for giving this particular rating in the previous question? [FREE TEXT]

\section{Your suggestions on new resources for LGBT+ young people}

At what age do you feel educational resources are needed on the topic of online safety for LGBT+ young people? Please rank in order of importance
a) Under 7
b) 7 to 11
c) 11 to 14
d) 14 to 16
e) Over 16

Should the resource we create be targeted at LGBT+ young people specifically or be adaptable for use with young people of any sexuality or gender?
a) Targeted at LGBT+ young people
b) Targeted at any sexuality or gender
c) Other

Please could you share any examples you can of good resources for LGBT+ young people? Examples do not need to be around the topic of online safety [FREE TEXT]

Is there anything else you feel we should consider as we begin to develop new resources for LGBT+ young people?

[FREE TEXT]

\section{Contact}

Would you be happy to be contacted further regarding your responses to this survey?

Yes

No 
Agnew-Brune, Christine B, Alexandra B Balaji, Brian Mustanski, Michael E Newcomb, Nik Prachand, Sarah L Braunstein, Kathleen A Brady, Brooke E Hoots, Justin S Smith, and Gabriela Paz-Bailey. 2019. "Mental health, social support, and HIV-related sexual risk behaviors among HIV-negative adolescent sexual minority males: three US cities, 2015." AIDS and Behavior 23 (12):3419-3426.

Albury, Kath, and Paul Byron. 2016. "Safe on my phone? Same-sex attracted young people's negotiations of intimacy, visibility, and risk on digital hook-up apps." Social Media+ Society 2 (4):1 - 10.

Alsaleh, K., Bragg, S., Gahnstrom, R., McGeeney, E., O'Riordan, K., and Thomson, R. (2020) Beyond digital romance: socialities, sexualities and online cultures for LGBTQ+ young people, Sussex Humanities Lab/ Centre for Innovation \& Research in Childhood \& Youth working paper. May 2020.

Alsaleh, Khaliden, Sara Bragg, Rosie Gahnstrom, Ester McGeeney, Kate O'Riordan, Rachel Thomson, Sussex Humanities Lab/, Centre for Innovation \& Research in Childhood 8 , and Youth working paper. May 2020. 2020. Beyond digital romance: socialities, sexualities and online cultures for LGBTQ+ young people, working paper. May 2020. Falmer, Sussex: Sussex Humanities Lab/ Centre for Innovation \& Research in Childhood \& Youth.

Andreassen, Rikke, Michael Nebeling Petersen, Katherine Harrison, and Tobias Raun. 2017. Mediated Intimacies: connectivities, relationalities and proximities. London and NY: Routledge.

Bain, Alison L., and Julie A. Podmore. 2020. "Morethan-safety: co-creating resourcefulness and conviviality in suburban LGBTQ2S youth out-ofschool spaces." Children's Geographies:1- 14.

Berriman, Liam, and Rachel Thomson. 2015. "Spectacles of intimacy? Mapping the moral landscape of teenage social media." Journal of Youth Studies 18 (5):583-597.
Bonell, Chris, Rebecca Meiksin, GJ Melendez-Torres, Peter Weatherburn, Charles Witzel, and Alec Miners. 2020. "How can e-health interventions reduce the 'syndemic' of HIV/STIs and sexual risk, substance use and mental ill health among men who have sex with men? Systematic review and evidence synthesis." PROSPERO 2018 CRD42018110317 Available from: https://www.crd.york.ac.uk/prospero/display_record. php? ID =CRD42018110317.

Bower-Brown, Susie, Sophie Zadeh, and Vasanti Jadva. 2021. "Binary-trans, non-binary and genderquestioning adolescents' experiences in UK schools." Journal of LGBT Youth:1-19.

Boyd, Danah. 2011. "Social network sites as networked publics: affordances, dynamics, and implications." In A networked self: identity, community, and culture on social network sites, edited by Z. Papacharissi, 47-66. New York and London: Routledge.

Boyd, Danah. 2014. It's Complicated: The Social Lives of Networked Teens. New Haven and London: Yale University Press.

Bragg, Sara, Emma Renold, Jessica Ringrose, and Carolyn Jackson. 2018. "'More than boy, girl, male, female': exploring young people's views on gender diversity within and beyond school contexts." Sex Education 18 (4):420-434.

Browne, Kath, Niharika Banerjea, Nick McGlynn, Leela Bakshi, Sumita Beethi, and Ranjita Biswas. 2021. "The limits of legislative change: Moving beyond inclusion/ exclusion to create 'a life worth living'." Environment and Planning C: Politics and Space 39 (1):30-52.

Bryan, Austin. 2019. "Kuchu activism, queer sex-work and "lavender marriages," in Uganda's virtual LGBT safe(r) spaces." Journal of Eastern African Studies 13 (1):90-105.

Byron, Paul. 2015. "Troubling expertise: social media and young people's sexual health." Communication Research and Practice 1 (4):322-334. 
Byron, Paul. 2017. "Friendship, sexual intimacy and young people's negotiations of sexual health." Culture, Health \& Sexuality 19 (4):486-500.

Byron, Paul. 2017. "The intimacies of young people's sexual health and pleasure." Journal of Youth Studies 20 (3):332-348.

Byron, Paul, and Jessie Hunt. 2017. "'That happened to me too': young people's informal knowledge of diverse genders and sexualities." Sex Education 17 (3):319-332.

Cho, Alexander. 2018. “Default publicness: Queer youth of color, social media, and being outed by the machine." New Media \& Society 20 (9):3183-3200.

Cover, Rob. 2012. "Performing and undoing identity online: social networking, identity theories and the incompatibility of online profiles and friendship regimes." Convergence 18 (2):177-193.

DeHaan, Samantha, Laura E. Kuper, Joshua C. Magee, Lou Bigelow, and Brian S. Mustanski. 2013. "The interplay between online and offline explorations of identity, relationships, and sex: a mixed-methods study with LGBT youth." The Journal of Sex Research 50 (5):421- 434.

DeNardis, Laura, and Andrea M. Hackl. 2016. "Internet control points as LGBT rights mediation." Information, Communication \& Society 19 (6):753-770.

Downing, Gary. 2013. "Virtual youth: nonheterosexual young people's use of the internet to negotiate their identities and socio-sexual relations." Children's Geographies 11 (1):44-58.

Duguay, Stefanie. 2016. "He has a way gayer Facebook than I do": Investigating sexual identity disclosure and context collapse on a social networking site." New Media \& Society 18 (6):891-907.

Fish, Jessica N., Lauren B. Mclnroy, Megan S. Paceley، Natasha D. Williams, Sara Henderson, Deborah S. Levine, and Rachel N. Edsall. 2020. "I'm kinda stuck at home with unsupportive parents right now": LGBTQ youths' experiences with COVID-19 and the importance of online support." Journal of Adolescent Health 67 (3):450-452.

Fraser, Nancy. 1990. "Rethinking the Public Sphere: A Contribution to the Critique of Actually Existing Democracy." Social Text (25/26):56-80.

Gabb, Jacqui, Elizabeth McDermott, Rachael Eastham, and Ali Hanbury. 2020. "Paradoxical family practices: LGBT+ young people, mental health and wellbeing." Journal of Sociology 56 (4):535-553.
Ging, D., and Eugenia S., eds. 2019. Gender hate online: Understanding the New Anti-feminism. Basingstoke: Palgrave Macmillan Springer.

Gray, Mary L. 2009 a. "Negotiating identities/queering desires: coming out online and the remediation of the coming-out story." Journal of Computer-Mediated Communication 14 (4):1162-1189.

Gray, Mary L. 2009 b. Out in the country: youth, media, and queer visibility in rural America. Vol. 2. London and NY: NYU Press.

Hanckel, Benjamin, and Shiva Chandra. 2021. Social media insights from sexuality and gender diverse young people during COVID-19. Sydney, Australia: Western Sydney University.

Hanckel, Benjamin, Son Vivienne, Paul Byron, Brady Robards, and Brendan Churchill. 2019. "'That's not necessarily for them': LGBTIQ+ young people, social media platform affordances and identity curation." Media, Culture \& Society 41 (8):1261-1278.

Harper, Gary, Pedro Serrano, Douglas Bruce, and Jose Bauermeister. 2015. "The internet's multiple roles in facilitating the sexual orientation identity development of gay and bisexual male adolescents." American journal of men's health 10.

Jenzen, Olu. 2017. "Trans youth and social media: moving between counterpublics and the wider web." Gender, Place \& Culture 24 (11):1626-1641.

Jenzen, Olu, and Irmgard Karl. 2014. "Make, share, care: Social media and LGBTQ youth engagement." Ada: A journal of gender, new media, and technology 5.

Jones, Tiffany. 2013. "How sex education research methodologies frame GLBTIQ students." Sex Education 13 (6):687-701.

Keller, Jessalynn. 2019. " "Oh, she's a Tumblr feminist": exploring the platform vernacular of girls' social media feminisms." Social Media + Society 5 (3):2056305119867442.

Latimer, Joanna, and Daniel López Gómez. 2019. "Intimate Entanglements: Affects, more-than-human intimacies and the politics of relations in science and technology." The Sociological Review 67 (2):247-263.

Marston, Kate. 2019. "Researching LGBT+ youth intimacies and social media: the strengths and limitations of participant-led visual methods." Qualitative Inquiry 25 (3):278-288. 
Marwick, Alice, and Danah Boyd. 2014. "'It's just drama': teen perspectives on conflict and aggression in a networked era." Journal of Youth Studies 17 (9):1187-1204.

McGeeney, Ester, and Elly Hanson. 2017. Digital Romance: a research project exploring young people's use of technology in their romantic relationships and love lives. London: National Crime Agency and Brook.

Miles, Sam. 2018. "Still getting it on online: thirty years of queer male spaces brokered through digital technologies." Geography Compass 12 (11):e12407.

Paceley, Megan S., Lance C. Keene, and Benjamin J. Lough. 2015. "Motivations for involvement in nonmetropolitan LGBTQ organizations: a multimethod qualitative exploration." Journal of Community Practice 23 (1):102-125.

Paceley, Megan S., Lance C. Keene, and Benjamin J. Lough. 2016. "Barriers to involvement in nonmetropolitan LGBTQ organizations." Journal of Gay \& Lesbian Social Services 28 (2):117-139.

Prock, Kristen A., and Angie C. Kennedy. 2017. "Federally-funded transitional living programs and services for LGBTQ-identified homeless youth: a profile in unmet need." Children and Youth Services Review 83:17-24.

Raun, Tobias. 2014. “Video blogging as a vehicle of transformation: Exploring the intersection between trans identity and information technology." International Journal of Cultural Studies 18 (3):365-378.

Renninger, Bryce J. 2015. "Where I can be myself ... where I can speak my mind": Networked counterpublics in a polymedia environment." New Media \& Society 17 (9):1513-1529.

Ringrose, Jessica, and Emma Renold. 2010. "Normative cruelties and gender deviants: the performative effects of bully discourses for girls and boys in school." British Educational Research Journal 36 (4):573-596.
Ringrose, Jessica, and Laura Harvey. 2017. "Digital mediation, connectivity and affective materialities." In Routledge Handbook of Physical Cultural Studies, edited by ML. Silk, DL. Andrews and H Thorpe. Abingdon UK: Routledge.

Selkie, Ellen, Victoria Adkins, Ellie Masters, Anita Bajpai, and Daniel Shumer. 2020. "Transgender adolescents' uses of social media for social support." Journal of Adolescent Health 66 (3):275-280.

Srnicek, Nick. 2017. Platform Capitalism, Theory Redux. Cambridge, UK: Polity. Book.

Vickery, Jacqueline Ryan. 2015. "'I don't have anything to hide, but...': the challenges and negotiations of social and mobile media privacy for non-dominant youth." Information, Communication \& Society 18 (3):281-294.

Warner, Michael. 1991. "Introduction: fear of a queer planet." Social Text (29):3-17.

Warner, Michael. 2002. "Publics and counterpublics (abbreviated version)." Quarterly Journal of Speech 88 (4):413-425.

Watson, Ash, Deborah Lupton, and Mike Michael. 2021. "Enacting intimacy and sociality at a distance in the COVID-19 crisis: the sociomaterialities of home-based communication technologies." Media International Australia 178 (1):136-150.

Wight, Jules. 2014. "Queer sweet home: disorientation, tyranny, and silence in digital space." Cultural Studies $\leftrightarrow$ Critical Methodologies 14 (2): 128-137.

Zuboff, Shoshana. 2019. The age of surveillance capitalism: the fight for a human future at the new frontier of power. NY: Profile Books. 


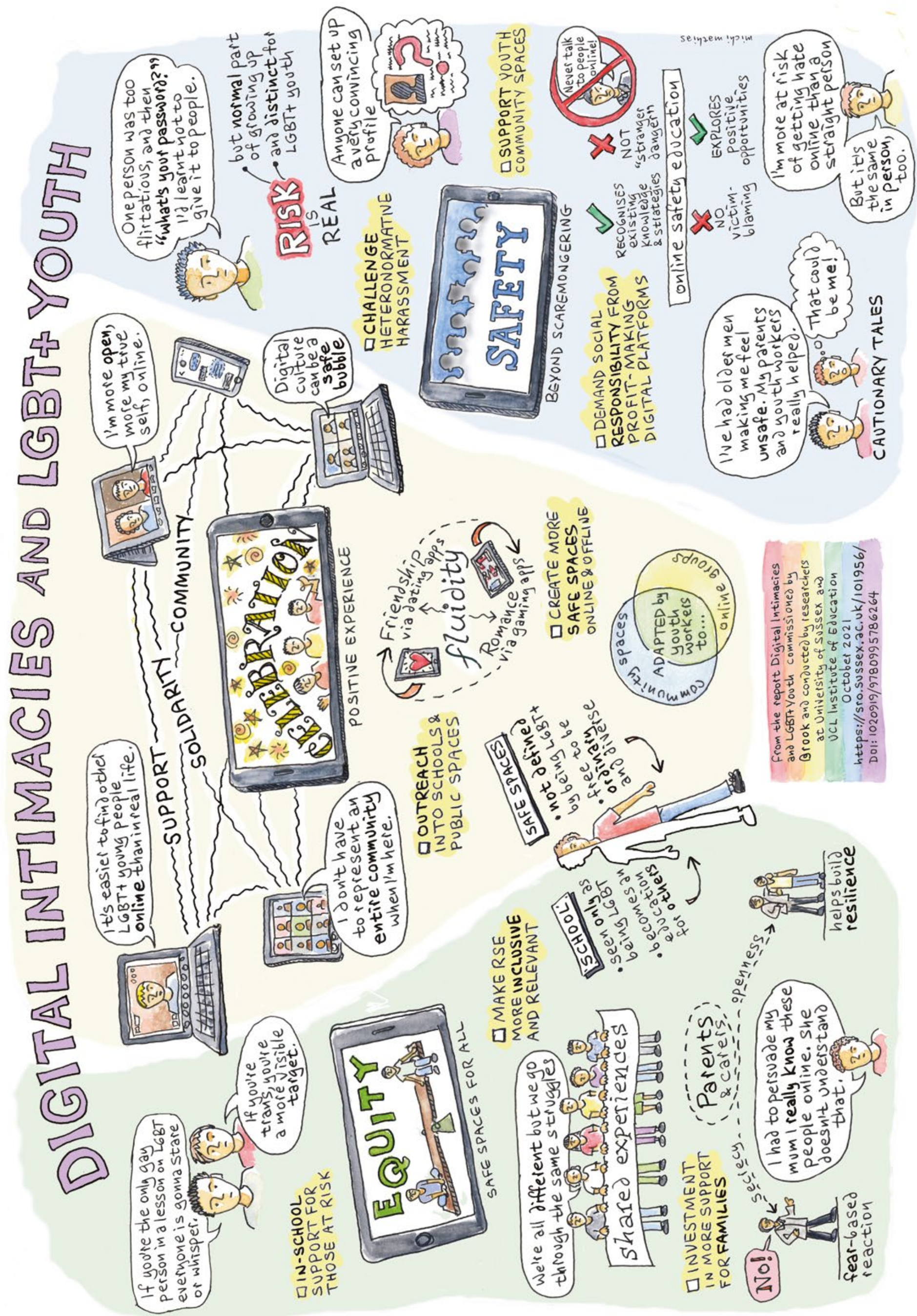

